\title{
Correction to: Quantifying the Collision Dose in Rugby League: A Systematic Review, Meta-analysis, and Critical Analysis
}

Mitchell Naughton ${ }^{1 *}$, Ben Jones ${ }^{1,2,3,4,5}$, Sharief Hendricks ${ }^{5,2,6}$, Doug King ${ }^{1,7,8}$, Aron Murphy ${ }^{1}$ and Cloe Cummins ${ }^{1,2,9}$

\author{
Correction to: Sports Med - Open 6:6 (2020) \\ https://doi.org/10.1186/s40798-019-0233-9
}

An incompletely revised version of the above article was inadvertently submitted for publication before all authors had the opportunity to approve the manuscript. As a result, multiple errors were present in the published article [1].

These relate to:

- The number of articles assessed.

- Reference citation details.

- Data errors.

- Figs 1, 2a, 2b, 3a, 3b, 4a, 4b, 5, 6a, 6b and 6c.

- Tables 1, 2, 3, 4 and 6 .

The full article with all corrections is re-published ahead.

\section{Author details}

${ }^{1}$ School of Science and Technology, University of New England, Armidale, NSW, Australia. ${ }^{2}$ Carnegie Applied Rugby Research (CARR) centre, Institute for Sport Physical Activity and Leisure, Leeds Beckett University, Leeds, UK. ${ }^{3}$ Leeds Rhinos Rugby League club, Leeds, UK. ${ }^{4}$ England Performance Unit, The Rugby Football League, Leeds, UK. ${ }^{5}$ Division of Exercise Science and Sports Medicine, Department of Human Biology, Faculty of Health Sciences, University of Cape Town, Cape Town, South Africa. ${ }^{6}$ Health through Physical Activity, Lifestyle and Sport Research Centre (HPALS), Faculty of Health Sciences, The University of Cape Town, Cape Town, South Africa. ${ }^{7}$ Sports Performance Institute New Zealand (SPRINZ), Faculty of Health and Environmental Science, Auckland University of Technology, Auckland, New Zealand. ${ }^{8}$ School of Sport, Exercise and Nutrition, Massey University, Palmerston North, New Zealand. ${ }^{9}$ National Rugby League, Sydney, Australia.

\section{Reference}

1. Naughton M, et al. Quantifying the Collision Dose in Rugby League: A Systematic Review, Meta-analysis, and Critical Analysis. Sports Med - Open. 2020;6:6. https://doi.org/10.1186/s40798-019-0233-9.

\footnotetext{
* Correspondence: mnaught2@myune.edu.au

The original article can be found online at https://doi.org/10.1186/s40798019-0233-9

${ }^{1}$ School of Science and Technology, University of New England, Armidale, NSW, Australia

Full list of author information is available at the end of the article
}

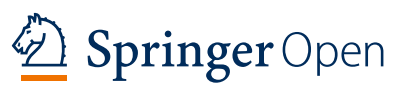

(c) The Author(s). 2020 Open Access This article is licensed under a Creative Commons Attribution 4.0 International License, which permits use, sharing, adaptation, distribution and reproduction in any medium or format, as long as you give appropriate credit to the original author(s) and the source, provide a link to the Creative Commons licence, and indicate if changes were made. The images or other third party material in this article are included in the article's Creative Commons licence, unless indicated otherwise in a credit line to the material. If material is not included in the article's Creative Commons licence and your intended use is not permitted by statutory regulation or exceeds the permitted use, you will need to obtain permission directly from the copyright holder. To view a copy of this licence, visit http://creativecommons.org/licenses/by/4.0/. 


\title{
Quantifying the collision dose in rugby league: a systematic review, meta-analysis, and critical analysis
}

Mitchell Naughton ${ }^{1 *}$, Ben Jones ${ }^{1,2,3,4,5}$, Sharief Hendricks ${ }^{5,2,6}$, Doug King ${ }^{1,7,8}$, Aron Murphy ${ }^{1}$ and Cloe Cummins $s^{1,2,9}$

\begin{abstract}
Background: Collisions (i.e. tackles, ball carries, and total collisions) in rugby league have the potential to increase injury risk, delay recovery, and influence individual and team performance. Understanding the collision demands of rugby league may enable practitioners to optimise player health, recovery, and performance.

Objective: The aim of this review was to: 1) characterise the dose of collisions experienced within senior male rugby league match-play and training; 2) evaluate the methods used to describe the relative and absolute frequency and intensity of collisions; and 3) provide recommendations on collision monitoring in rugby league.

Methods: A systematic search of electronic databases (PubMed, SPORTDiscus, Scopus, and Web of Science) using keywords was undertaken. A meta-analysis provided a pooled mean of collision frequency or intensity metrics on comparable data sets.
\end{abstract}

Results: Thirty-eight articles addressing the absolute $(n)$ or relative collision frequency $\left(n \cdot \mathrm{min}^{-1}\right)$ or intensity of senior male rugby league collisions were included. Meta-analysis of video-based studies identified that forwards completed approximately twice the number of tackles per game than backs ( $n=24.9$ vs 12.7), whilst ball carry frequency remained similar between backs and forwards ( $n=11.4 \mathrm{vs} 11.2$ ). Variable findings were observed at the subgroup level with a limited number of studies suggesting wide-running forwards, outside backs, and hit-up forwards complete similar ball carries whilst tackling frequency differed. For microtechnology, at the team level, players complete an average of 32.7 collisions per match. Limited data suggested hit-up and wide-running forwards complete the most collisions per match, when compared to adjustables and outside backs. Relative to playing time, forwards $\left(n \cdot \mathrm{min}^{-1}=0.44\right)$ complete a greater frequency of tackles than backs $\left(n \cdot \mathrm{min}^{-1}=0.16\right)$, with data suggesting hit-up forwards undertake more than adjustables, and outside backs. Studies investigating $g$ force intensity zones utilised four unique intensity schemes with zones ranging from 2-3 to $>12 \mathrm{~g}$. Given the disparity in device setups and zone classification systems between studies, further analyses were inappropriate. It is recommended that practitioners independently validate microtechnology against video to establish criterion validity.

(Continued on next page)

\footnotetext{
* Correspondence: mnaught2@myune.edu.au

${ }^{1}$ School of Science and Technology, University of New England, Armidale,

NSW, Australia

Full list of author information is available at the end of the article
}

\section{Springer Open}

(c) The Author(s). 2020 Open Access This article is licensed under a Creative Commons Attribution 4.0 International License, which permits use, sharing, adaptation, distribution and reproduction in any medium or format, as long as you give appropriate credit to the original author(s) and the source, provide a link to the Creative Commons licence, and indicate if changes were made. The images or other third party material in this article are included in the article's Creative Commons licence, unless indicated otherwise in a credit line to the material. If material is not included in the article's Creative Commons licence and your intended use is not permitted by statutory regulation or exceeds the permitted use, you will need to obtain permission directly from the copyright holder. To view a copy of this licence, visit http://creativecommons.org/licenses/by/4.0/. 
(Continued from previous page)

Conclusions: Video- and microtechnology-based methods have been utilised to quantify collisions in rugby league with differential collision profiles observed between forward and back positional groups, and their distinct subgroups. The ball carry demands of forwards and backs were similar, whilst tackle demands were greater for forwards than backs. Microtechnology has been used inconsistently to quantify collision frequency and intensity. Despite widespread popularity in rugby league, a number of the microtechnology devices have yet to be appropriately validated. Limitations exist in using microtechnology to quantify collision intensity, including the lack of consistency and limited validation. Future directions include application of machine learning approaches to differentiate types of collisions in microtechnology datasets.

\section{Key Points}

- Video-, and microtechnology-based methods have been employed to quantify collision (including tackle and ball carry) frequency and intensity with position-specific differences observed.

- A number of microtechnology devices that purport collision detection capacity have yet to be appropriately validated, and practitioners should be aware of these limitations when utilising such devices to quantify collisions.

- There are considerable gaps in the understanding of effectively quantifying collisions in rugby league, which may be explored by applying machine learning methods to microtechnology datasets.

\section{Introduction}

Rugby league is an invasion contact sport played in over 14 countries, in which senior male rugby league consists of two 40 minute halves [1]. A match is contested by 13 players on two opposing teams. The fundamental goal of rugby league is to score more points than the opposition team and this can be achieved by scoring a try (i.e. grounding the ball beyond the oppositions try line) or kicking a goal (i.e. a drop goal, penalty kick, or try conversion) [2]. Whilst the demands of rugby league are specific to the respective competition [3], playing level [4], and positional group [5], the game typically involves intermittent periods of low intensity exercise (such as walking or jogging), interspersed with periods of highintensity efforts (such as accelerations, decelerations, running, and sprinting) [6-9].

Global Positioning Systems (GPS) are an accurate satellite-based navigational technology that was first launched in 1978 [10-12]. Commercial GPS devices were first utilised within sporting contexts in 1997 [13]. Upon their introduction, these devices sampled at $1 \mathrm{Hertz}(\mathrm{Hz})$ with limited accompanying software [13]. Such devices vices have however, evolved over time to include higher sampling rates (e.g. 5 or $10 \mathrm{~Hz}$ ) and custom proprietary local software and cloud-based computing [14]. Alongside this evolution, additional inertial sensors such as accelerometers, gyroscopes, and magnetometers have been incorporated into these devices [15]. These sensors provide information on the instantaneous rate of accelerations in the $x$-, $y$-, and $z$-axis (i.e. anteroposterior, mediolateral, and vertical), as well as yaw, pitch, and roll, and unit orientation in relation to the earth's magnetic poles [10]. The combination of GPS and imbedded inertial sensors is referred to as a microtechnology device. Utilising microtechnology in sporting contexts, research has examined a variety of variables including work rate patterns, movement profiles, and the peak locomotor demands of training and competition in sports such as soccer [16-19], Australian rules [16, 20, 21], rugby union $[22,23]$, and rugby league $[4,15,24,25]$.

Microtechnology devices were first introduced into professional rugby league via the National Rugby League (NRL) and European Super League (SL) in 2009 and 2010, respectively [9]. Since their introduction, there has been an increase in the research utilising these devices to monitor match-play and training demands. A PubMed search of all studies published between 2009 and 2019 using the terms "Rugby League AND GPS" identified an increase from one article in 2010, to 19 published in 2018 and 2019. The locomotor demands of rugby league have been previously described in detail $[4,9]$. Additionally, rugby league is characterised by collisions between teammates and their opponents [26], as well as the playing surface. These collisions typically occur between the tackler(s) and the ballcarrier during the tackle event, and have been reported to lead to soreness and muscle damage which compromises muscle integrity, attenuates force generation capacity, and this has the potential to delay athletic recovery [27]. Additionally, the vast majority ( 94\%) of match-related injuries in professional rugby league are tackle related [28]. Furthermore, dominance in collision events has been shown to relate to match performance (i.e. match outcome) from both attacking and defending perspectives [29-31]. Given the apparent importance of offensive (e.g. ball carries) and defensive collisions (e.g. tackles) to match outcome and player health and wellbeing, it is imperative for coaches and practitioners to specifically monitor the collision demands of both training and competition activities.

Historically, quantification of the volume and intensity of collisions experienced (i.e. 'dose') by rugby league 
athletes have occurred via tallies of tackles and ballcarries, and through qualitative examination of the perceived dominance in collisions from analysis of video footage [32, 33]. While this process can provide a rich source of contextual data, it is often labor and resource intensive, and may be prone to the subjective biases of the video analyst [31]. Furthermore, the time taken to analyse these activities can be problematic due to the limited turn-around between matches and training sessions. To address this, microtechnology has been utilised to automate the assessment of impacts and collisions based on changes in unit orientation through proprietary algorithms [34, 35]. Indeed, a number of microtechnology devices now have automated impact and collision detection capacity from companies including Catapult Sports (Catapult Sports, Melbourne,Victoria, Australia), STATSports (STATSports, Newry, Northern Ireland), and GPSports (GPSports, Canberra, Australian Capital Territory, Australia). Similarly, microtechnology have been utilised to quantify collision intensity through summating the forces acting upon the accelerometer into $g$ force intensity zones. However, the validity of these approaches in quantifying both collision frequency and intensity through microtechnology is unclear. Furthermore, an understanding of the collision dose experienced in male senior rugby league has yet to be fully elucidated. Given the rapid commercial development in this area, and the importance of quantifying collisions, this systematic review characterises the dose of collisions experienced within senior rugby league training and match activities and examines the utility of microtechnology devices in quantifying collisions. Therefore, in relation to male senior rugby league, the specific aims of this review were to: 1) evaluate the methods used to describe the relative and absolute frequency of collisions; 2) evaluate the methods used to describe the relative and absolute intensity of collisions; 3 ) collate the collision demands of match-play and training; and 4) critically examine the literature, and provide recommendations on the monitoring of collision loads in rugby league.

\section{Methods}

\section{Design}

Studies investigating the collision dose experienced by male senior rugby league athletes (i.e. athletes over 18 years of age) in training and match activities or game simulation were eligible for inclusion. A systematic search of electronic databases (PubMed, SPORTDiscus, Scopus, and Web of Science) were conducted from January 1990 to March 2019. The search strategy combined terms for collisions ('tackl"', OR 'collision', OR 'impact"'), AND dose ('frequency', OR 'intensity', OR 'demands'), AND rugby league ('rugby"', OR 'rugby league'). Any study that examined the frequency (number of collisions), intensity or the type of collision (such as impacts, collisions, tackles, ball-carries) in a quantitative manner were included.

\section{Selection of studies}

Following the elimination of duplicate manuscripts, the search results were parsed for eligibility by examination of the title and abstract by one of the researchers $(\mathrm{MN})$. References that could be eliminated by title or abstract examination were removed and the remaining studies were screened by two researchers $(\mathrm{MN}, \mathrm{CC})$ against the eligibility criteria. Screening occurred via a customised spreadsheet, and there were no disagreements in the included studies between researchers. Reviewers were not masked to the names of authors or the title of publications. Abstracts and conference papers from annual meetings were not included due to not meeting the rigor of outcome measures. In instances where journal articles contained insufficient information, attempts were made to contact the authors in order to obtain further details, with one paper being excluded due to data not being made available to the authors on request [36]. Papers from all languages were included but were excluded if translation to English could not be made. Reference lists of papers included in the final analysis were screened for inclusion of other potentially eligible papers as included from alternate sources' (see Fig. 1).

\section{Data extraction}

Data relating to the participant characteristics (i.e. age, height, body mass, and competition level), the method used to quantify collisions (i.e. video or microtechnology), microtechnology device (i.e. model, manufacturer, recording frequency, presence of inertial sensors), collision characteristics, frequency of collisions, and the intensity of collisions were extracted. Collision characteristics included what was reported with respect to impacts, collisions, or differentiated into player tackle or ball carry into contact. The frequency of collisions were extracted as the absolute number $(n)$ per match/simulation/training session at either the team, season, or competition level. Furthermore, collisions relative to playing or training time $\left(n \cdot \mathrm{min}^{-1}\right)$ were extracted. The intensity of collisions were extracted from studies which provided mild, moderate, or heavy descriptors based on the nature of the event [31], with these categories based on microtechnology data. The absolute and relative frequencies within these classifications were also extracted. Similarly, the relative and absolute frequency of collision forces ( $g$ forces) were divided into 4 to 6 different zones (zones 1-6) ranging from 2-3 to $>12 g[37,38]$. Each zone was linked to a qualitative description ranging from light impacts and change of direction, through to severe impacts and player collisions [4]. Velocity and acceleration into contact were also extracted 


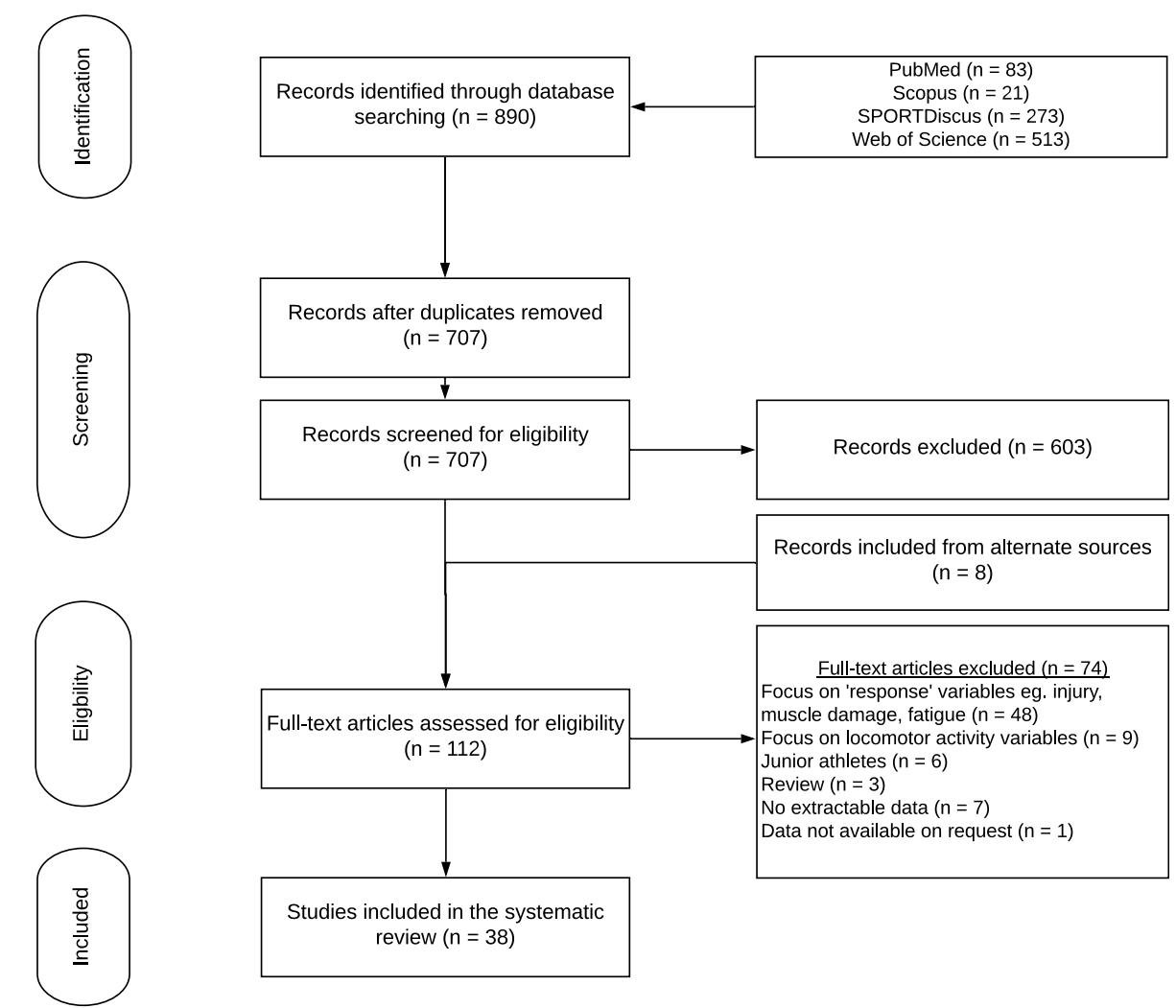

Fig. 1 Selection process of eligible studies in the systematic review

as a collision pseudo-intensity metric. Data which were only available in graphical form were extracted by digitising of the figures with WebPlotDigitizer [39].

\section{Assessment of methodological quality}

The quality of the included studies was independently assessed using the modified assessment scale of Downs and Black [40]. Of the 27 criteria, 12 questions were examined that logically applied to the study designs utilising microtechnology or video analysis in sport. These criteria questions reflected subscales that relate to external validity (numbers 11,12), internal validity $(16,18,20)$, and reporting $(1-4,6,7,10)$. Assessment of quality was completed by two of the authors (MN, CC). No studies were eliminated, and no additional subgroup analysis was undertaken on the basis of methodological quality.

\section{Statistics}

All data are presented as mean or mean \pm standard deviation (SD) unless otherwise stated. Where possible data that were published as mean and associated confidence intervals were transformed to SD [25, 41, 42] utilising methods outlined in the Cochrane handbook [43]. Where this was not possible due to insufficient information ( $n=1$ study [44]), data were reported as mean and relevant confidence intervals. Studies were included in the meta-analysis if they reported the number of player files or the number of participants. Meta-analyses (Review Manager, Version 5.3) were conducted to provide a pooled mean with 95\% confidence intervals for collision dose of the groups and subgroups for which comparable data were extracted from at least two similar studies. Meta-analysis was not undertaken on grouped or sub-grouped data when there were insufficient data to compare between studies for a given group or subgroup comparison. For consistency, studies were entered into the meta-analysis if they reported the number of players as the sample size, and SD or standard error (SE) was reported, or if those values could be derived from the available information within the manuscript. Studies that were not entered into the meta-analysis are presented in tables and/or text. When studies reported data from different cohorts within the same study, these were treated as data from separate studies [45]. Heterogeneity of studies within-, and between-subgroups was assessed via Chi-squared $\left(\mathrm{Chi}^{2}\right)$, Tau-squared $\left(\mathrm{Tau}^{2}\right)$ and I-squared $\left(I^{2}\right)$ statistics [43]. An $I^{2}$ of $0-40 \%, 40-75 \%$, and $>75 \%$ was considered low, moderate, and high heterogeneity respectively [43]. The following variables were included in the metaanalysis; positional group, absolute collision frequency $(n)$, relative collision frequency $\left(n \cdot \mathrm{min}^{-1}\right)$, absolute collision intensity (mild/moderate/heavy) and the type of collision reported (tackle/ball carry/collisions). 
Table 1 Methodological quality assessment of the included studies (Downs and Black 1998 [40])

\begin{tabular}{|c|c|c|c|c|c|c|c|c|c|c|c|c|}
\hline \multirow[t]{2}{*}{ Study } & \multicolumn{11}{|c|}{ Question Number } & \multirow{2}{*}{$\begin{array}{l}\text { Total } \\
\text { Score }\end{array}$} \\
\hline & 1 & 2 & 3 & 6 & 7 & 10 & 11 & 12 & 16 & 18 & 20 & \\
\hline Austin et al. [46] & 1 & 1 & 0 & 1 & 0 & 0 & $0^{a}$ & 0 & 1 & 1 & 1 & 6 \\
\hline Cummins \& Orr [60] & 1 & 1 & 0 & 1 & 0 & 1 & 1 & 1 & 1 & 1 & 1 & 9 \\
\hline Cummins \& Orr [26] & 1 & 1 & 1 & 1 & 1 & 1 & $0^{a}$ & 0 & 1 & 1 & 1 & 9 \\
\hline Dempsey et al. [8] & 1 & 1 & 1 & 1 & 1 & 0 & $0^{a}$ & 1 & 1 & 1 & 1 & 9 \\
\hline Evans et al. [65] & 1 & 1 & 1 & 1 & 1 & 1 & $0^{\mathrm{a}}$ & 0 & 1 & 1 & 1 & 9 \\
\hline Fletcher et al. [66] & 1 & 1 & 1 & 1 & 1 & 1 & $0^{\mathrm{a}}$ & 0 & 1 & 1 & 1 & 9 \\
\hline Gabbett et al. [31] & 1 & 1 & 1 & 1 & 1 & 0 & $0^{a}$ & 0 & 1 & 1 & 1 & 8 \\
\hline Gabbett \& Ryan [67] & 1 & 1 & 1 & 1 & 1 & 1 & $0^{a}$ & 0 & 1 & 1 & 1 & 9 \\
\hline Gabbett [33] & 1 & 1 & 1 & 1 & 1 & 1 & $0^{a}$ & 0 & 1 & 1 & 1 & 9 \\
\hline Gabbett [24] & 0 & 1 & 1 & 1 & 1 & 1 & $0^{\mathrm{a}}$ & 0 & 1 & 1 & 1 & 8 \\
\hline Gabbett [47] & 1 & 1 & 1 & 1 & 1 & 1 & 1 & 1 & 1 & 1 & 1 & 11 \\
\hline Gabbett [48] & 1 & 1 & 1 & 1 & 1 & 1 & 1 & 1 & 1 & 1 & 1 & 11 \\
\hline Gabbett et al. [44] & 1 & 1 & 1 & 1 & 1 & 0 & $0^{a}$ & 0 & 1 & 1 & 1 & 8 \\
\hline Gabbett et al. [49] & 1 & 1 & 1 & 1 & 1 & 0 & $0^{a}$ & 0 & 1 & 1 & 1 & 8 \\
\hline Gabbett et al. [25] & 1 & 1 & 1 & 1 & 1 & 1 & $0^{\mathrm{a}}$ & 0 & 1 & 1 & 1 & 9 \\
\hline Gabbett \& Seibold [50] & 1 & 1 & 1 & 1 & 1 & 1 & $0^{a}$ & 0 & 1 & 1 & 1 & 9 \\
\hline Gabbett et al. [51] & 1 & 1 & 1 & 1 & 1 & 1 & $0^{a}$ & 0 & 0 & 1 & 1 & 8 \\
\hline Hulin et al. [35] & 1 & 1 & 1 & 1 & 1 & 1 & $0^{a}$ & 0 & 1 & 1 & 1 & 9 \\
\hline Hulin et al. [52] & 1 & 1 & 1 & 1 & 1 & 1 & $0^{a}$ & 1 & 1 & 1 & 1 & 10 \\
\hline Johnston et al. [53] & 1 & 1 & 1 & 1 & 1 & 1 & $0^{a}$ & 0 & 1 & 1 & 1 & 9 \\
\hline Kempton et al. [54] & 1 & 1 & 1 & 1 & 1 & 1 & $0^{a}$ & 0 & 1 & 1 & 1 & 9 \\
\hline Kempton et al. [42] & 1 & 1 & 1 & 1 & 1 & 1 & $0^{\mathrm{a}}$ & 1 & 1 & 1 & 1 & 10 \\
\hline King et al. [41] & 1 & 1 & 0 & 1 & 1 & 1 & 1 & 1 & 1 & 1 & 1 & 10 \\
\hline King et al. [28] & 1 & 1 & 0 & 1 & 1 & 1 & $0^{a}$ & 0 & 1 & 1 & 1 & 8 \\
\hline Lovell et al. [55] & 1 & 1 & 1 & 1 & 1 & 1 & $0^{a}$ & 0 & 1 & 1 & 1 & 9 \\
\hline McLellan \& Lovell [56] & 1 & 1 & 1 & 1 & 1 & 1 & $0^{a}$ & 0 & 1 & 1 & 1 & 9 \\
\hline McLellan et al. [38] & 1 & 1 & 1 & 1 & 1 & 1 & $0^{a}$ & 0 & 1 & 1 & 1 & 9 \\
\hline Murray et al. [57] & 1 & 1 & 1 & 1 & 1 & 1 & $0^{\mathrm{a}}$ & 0 & 1 & 1 & 1 & 9 \\
\hline Oxendale et al. [58] & 1 & 1 & 1 & 1 & 1 & 1 & $0^{a}$ & 0 & 1 & 1 & 1 & 9 \\
\hline Sirotic et al. [32] & 1 & 1 & 1 & 1 & 1 & 1 & $0^{a}$ & 0 & 1 & 1 & 1 & 9 \\
\hline Sirotic et al. [59] & 1 & 1 & 1 & 1 & 1 & 1 & $0^{a}$ & 0 & 1 & 1 & 1 & 9 \\
\hline Speranza et al. [61] & 1 & 1 & 1 & 1 & 1 & 1 & $0^{\mathrm{a}}$ & 0 & 1 & 1 & 1 & 9 \\
\hline Twist et al. [62] & 1 & 1 & 1 & 1 & 1 & 1 & $0^{a}$ & 0 & 1 & 1 & 1 & 9 \\
\hline Varley et al. [16] & 1 & 1 & 0 & 1 & 1 & 1 & $0^{a}$ & 0 & 1 & 1 & 1 & 8 \\
\hline Weaving et al. [63] & 1 & 1 & 1 & 1 & 1 & 1 & $0^{a}$ & 0 & 1 & 1 & 1 & 9 \\
\hline Weaving et al. [7] & 1 & 1 & 1 & 1 & 1 & 1 & $0^{a}$ & 0 & 1 & 1 & 1 & 9 \\
\hline Woods et al. [1] & 1 & 1 & 0 & 1 & 1 & 1 & 1 & 1 & 1 & 1 & 1 & 10 \\
\hline Woods et al. [64] & 1 & 1 & 0 & 1 & 1 & 1 & 1 & 1 & 1 & 1 & 1 & 10 \\
\hline
\end{tabular}

1. Is the hypothesis/aim clearly described? 2. Are the main outcomes to be measured clearly described in the introduction/methods sections? 3 . Are the characteristics of the participants included in the study clearly described? 6 . Are the main findings of the study clearly described? 7. Does the study provide estimates of the variability in the data for the main outcome? 10. Have $p$ values/effect sizes for the main outcome been reported? 11. Were the subjects who were asked to participate representative of the wider population of interest? 12. Were the subjects who were prepared to participate representative of the wider population of interest? 16. Were any of the results based on 'data dredging', was this made clear? 18. Were the statistical tests used for the main outcomes appropriate? 20. Were the main outcome measures used accurate and reliable? ${ }^{a}$ Unable to determine 
Table 2 Study characteristics of the included studies

\begin{tabular}{|c|c|c|c|}
\hline Study & Method of collision capture & Level of Competition & No. of participants $(n=)$ \\
\hline Austin et al. [46] & Video & NRL & 15 \\
\hline Cummins \& Orr [60] & Video and microtechnology & NRL & $\begin{array}{l}\text { NR (Video) } \\
10 \text { (Microtechnology) }\end{array}$ \\
\hline Cummins \& Orr [26] & Video and microtechnology & NRL & 26 \\
\hline Dempsey et al. [8] & Video & International & 57 \\
\hline Evans et al. [65] & Microtechnology & Super League & 33 \\
\hline Fletcher et al. [66] & Video & Super League & 31 \\
\hline Gabbett et al. [31] & Video and microtechnology & NRL & 30 \\
\hline Gabbett \& Ryan [67] & Video & $\begin{array}{l}\text { NRL } \\
\text { QCup }\end{array}$ & $\begin{array}{l}22 \\
17\end{array}$ \\
\hline Gabbett [33] & Video & Local & 8 \\
\hline Gabbett [24] & Microtechnology & $\begin{array}{l}\text { NRL } \\
\text { NYC }\end{array}$ & $\begin{array}{l}24 \\
11\end{array}$ \\
\hline Gabbett [47] & Microtechnology & QCup & 182 \\
\hline Gabbett [48] & Microtechnology & QCup & 104 \\
\hline Gabbett et al. [44] & Video & NRL & 51 \\
\hline Gabbett et al. [49] & Video & NRL & 58 \\
\hline Gabbett et al. [25] & Microtechnology & NRL & 30 \\
\hline Gabbett \& Seibold [50] & Microtechnology & QCup & 32 \\
\hline Gabbett et al. [51] & Microtechnology & NRL & 38 \\
\hline Hulin et al. [35] & Video and microtechnology & NRL & 8 \\
\hline Hulin et al. [52] & Microtechnology & NRL & 31 \\
\hline Johnston et al. [53] & Microtechnology & International Student Competition & 7 \\
\hline Kempton et al. [54] & Microtechnology & NRL & 18 \\
\hline Kempton et al. [42] & Microtechnology & NRL & $\begin{array}{l}29 \text { (more successful) } \\
25 \text { (less successful) }\end{array}$ \\
\hline King et al. [41] & Video & $\begin{array}{l}\text { International } \\
\text { NRL }\end{array}$ & $\begin{array}{l}N R \\
N R\end{array}$ \\
\hline King et al. [28] & Video & NRL & NR \\
\hline Lovell et al. [55] & Microtechnology & NRL & 32 \\
\hline McLellan \& Lovell [56] & Video and microtechnology & NRL & 22 \\
\hline McLellan et al. [38] & Video and microtechnology & NRL & 17 \\
\hline Murray et al. [57] & Microtechnology & NRL & 43 \\
\hline Oxendale et al. [58] & Microtechnology & Super League & 17 \\
\hline Sirotic et al. [32] & Video & $\begin{array}{l}\text { NRL } \\
\text { NSWCup }\end{array}$ & $\begin{array}{l}17 \\
22\end{array}$ \\
\hline Sirotic et al. [59] & Video & NRL & 17 \\
\hline Speranza et al. [61] & Video & QCup & 16 \\
\hline Twist et al. [62] & Video & Super League & 23 \\
\hline Varley et al. [16] & Microtechnology & NRL & 36 \\
\hline Weaving et al. [63] & Microtechnology & Super League & 17 \\
\hline Weaving et al. [7] & Microtechnology & Super League & 25 \\
\hline Woods et al. [1] & Video & $\begin{array}{l}\text { NRL } \\
\text { Super League }\end{array}$ & $\begin{array}{l}\text { NR } \\
\text { NR }\end{array}$ \\
\hline Woods et al. [64] & Video & $\begin{array}{l}\text { NRL } \\
\text { NYC }\end{array}$ & $\begin{array}{l}\text { NR } \\
\text { NR }\end{array}$ \\
\hline
\end{tabular}




\section{Results}

\section{Identification and selection of studies}

The original search captured 890 papers (see Fig. 1). After the removal of duplicates and screening 38 studies were included in the systematic review $[1,7,8,16,24-$ 26, 28, 31-33, 35, 38, 41, 42, 44, 46-67].

\section{Methodological quality}

There were 38 studies that met the inclusion criteria. The methodological quality of these studies was moderate to good, with scores ranging from 6 to 11 across the 11 items that were assessed (see Table 1).

\section{Study characteristics}

Of the included manuscripts, four did not report on the number of participants. Collectively 1201 participants were examined ( $n=34$ studies). From these studies, participants were drawn from teams in the NRL, SL, Australian State Leagues (predominantly the Queensland Cup [QCup]), International, Australian Under 20's National Youth Competition (NYC), and amateur competitions (see Table 2).

Studies typically compared the collision dose either at the overall team level or within positional groupings. Analyses undertaken within positional groups included either two (backs and forwards [8, 24, 28, 38, 41, 47, 50, $56,58,61,62]$ ), or four (hit-up forwards [props], widerunning forwards [second-rowers, locks], adjustables [full-back, five-eighth, half-back, and hooker], and outside backs [wingers and centers] [25, 26, 28, 31, 41, 44, $52,65,66])$ positional groups. Studies reported collision dose features such as the absolute frequency of collisions per match $(n)$, collisions relative to a player's time on field $\left(n \cdot \mathrm{min}^{-1}\right)$, or collision intensity features that were derived from analysis of video footage (see Tables 2 and 3). Furthermore, studies reported features derived from microtechnology alone (see Tables 2 and 4) or via both methods (see Tables 2, 3 and 4).

Microtechnology devices from two manufacturers were utilised across eight studies (see Table 5) with four different $g$ force zone systems utilised by summating the tri-axial accelerometer force into zones 1 to 6. Microtechnology studies reported data according to intensity (i.e. mild, moderate or heavy) (see Table 4), or the absolute and relative force of collisions (as measured via $g$ force) across individual thresholds (e.g. zones 1 through to 6) (see Tables 5 and 6). A number of these studies $(n=5)$ utilised an impact metric for these zones that encompasses all forces acting on the accelerometer including from actions other than collisions (see Table 6) [38, 55, $56,63,65]$.

\section{Collision frequency \\ Video notational analysis}

A pooled analysis of these studies identified that forwards completed approximately twice the number of tackles per game than backs $(n=24.9$ vs 12.7 per match, $I^{2}=98.3 \%$ ) (see Fig. 2a), whilst the average number of ball carries remained relatively similar between forwards and backs ( $n=11.4$ vs 11.2 per match, $I^{2}=0 \%$ ) (see Fig. 3a). There were positional subgroup differences $\left(I^{2}=90.6 \%\right.$; see Fig. $\left.2 \mathrm{~b}\right)$, with the hit-up forwards ( $n=$ 22.4) undertaking a greater number of tackles per match than the adjustables $(n=14.8)$ and outside backs $(n=$ 7.4). Heterogeneity within positional groups was low for adjustables, hit-up forwards, and outside backs $\left(I^{2}<40 \%\right)$. Two studies found wide-running forwards complete a similar number of tackles per match as hit-up forwards [26, 44], but insufficient variance information was available in one study [44].

Conversely, both backs and forwards completed similar ball carries per match (see Fig. 3a). This result was mirrored when examining the positional subgroups with outside backs $(n=10.4)$, and hit-up forwards $(n=8.7)$ completing a somewhat similar number of ball carries per match (see Fig. 3b). However, adjustables $(n=4.2)$ undertook considerably fewer ball carries per match than all other positional groups (see Fig. 3b). Two studies found wide-running forwards complete an average frequency of ball carries per match which is similar to outside backs ( $n=7.9-17.0)$ [26, 44], but there was insufficient data relating to the variance of findings in one study [44].

Pooled analysis identified that forwards undertook a greater relative frequency of tackles per match when compared to backs $\left(n \cdot \mathrm{min}^{-1}=0.44\right.$ vs. $\left.0.16, I^{2}=98.6 \%\right)$ (see Fig. 4a). Relative ball carry frequency was also higher in the forwards when compared to backs $\left(n \cdot \min ^{-1}=0.25\right.$ vs. $\left.0.11, I^{2}=94.0 \%\right)$ (see Fig. $\left.4 \mathrm{~b}\right)$.

At the competition level, SL teams completed more ball carries per match than NRL teams, with a relatively similar tackle load [1], whilst NRL teams completed a greater number of tackles and ball carries when compared to their NYC counterparts [64]. Finally, international matches played at the Rugby League World Cup and the NRL competition standard contain a similar frequency of tackles and ball carries [41], with forwards and backs undertaking a greater number of tackles and ball carries respectively, at both the international and NRL levels [41].

\section{Microtechnology}

For those studies that utilised microtechnology to quantify collisions, pooled analysis identified 32.7 collisions per match with high heterogeneity $\left(I^{2}=98.0 \%\right)$ (see Fig. 5 ).

Studies using microtechnology reported that forwards undertook between 18.3 and 44.0 collisions per match on average, whilst one study reported that backs undertook 
Table 3 Characteristics of collisions during match-play recorded by video notational analysis

\begin{tabular}{|c|c|c|c|c|c|}
\hline Study & Competition (season[s]) & Positional Group & Type of collision recorded & $\begin{array}{l}\text { Frequency of } \\
\text { collisions }(n=) \\
\text { mean }( \pm \text { SD) }\end{array}$ & $\begin{array}{l}\text { Relative frequency } \\
\text { of collisions }\left(n \cdot \mathrm{min}^{-1}=\right) \\
\text { mean }( \pm \text { SD) }\end{array}$ \\
\hline \multirow[t]{3}{*}{ Austin et al. [46] } & NRL (2008) & Hit-up forwards & Tackles and ball carries & 33.2 (NR) & NR \\
\hline & & Adjustables & Tackles and ball carries & 17.8 (NR) & NR \\
\hline & & Outside backs & Tackles and ball carries & 8.2 (NR) & NR \\
\hline \multirow[t]{12}{*}{ Cummins \& Orr [26] } & $N R L(N R)$ & Hit-up forwards & Tackles & $21.5(6.1)$ & $0.52(0.09)$ \\
\hline & & & Ball carries & $8.9(3.7)$ & $0.20(0.03)$ \\
\hline & & & Tackles and ball carries & $30.5(9.6)$ & $0.78(0.11)$ \\
\hline & & Wide-running forwards & Tackles & $20.6(5.0)$ & $0.39(0.10)$ \\
\hline & & & Ball carries & $7.9(3.7)$ & $0.20(0.10)$ \\
\hline & & & Tackles and ball carries & $29.8(6.2)$ & $0.57(0.20)$ \\
\hline & & Adjustables & Tackles & $16.7(12.8)$ & $0.41(0.20)$ \\
\hline & & & Ball carries & $4.9(4.6)$ & $0.10(0.00)$ \\
\hline & & & Tackles and ball carries & $21.7(12.3)$ & $0.49(0.20)$ \\
\hline & & Outside backs & Tackles & $7.0(6.1)$ & $0.08(0.07)$ \\
\hline & & & Ball carries & $11.2(2.0)$ & $0.10(0.02)$ \\
\hline & & & Tackles and ball carries & $18.3(5.4)$ & $0.21(0.06)$ \\
\hline \multirow[t]{4}{*}{ Dempsey et al. [8] } & SL (2011-2012) & Backs & Tackles & $13.4(9.5)$ & $0.16(0.11)$ \\
\hline & & & Ball carries & $11.9(5.2)$ & $0.15(0.08)$ \\
\hline & & Forwards & Tackles & $25.5(8.4)$ & $0.47(0.23)$ \\
\hline & & & Ball carries & $10.5(3.6)$ & $0.20(0.10)$ \\
\hline \multirow[t]{9}{*}{ Fletcher et al. [66] } & SL (2012) & Hit-up forwards & Tackles & $24.0(13.0)$ & NR \\
\hline & & & Ball carries & $8.5(5.0)$ & NR \\
\hline & & & Tackles and ball carries & $32.0(15.0)$ & $0.60(0.30)$ \\
\hline & & Adjustables & Tackles & $14.0(12.0)$ & NR \\
\hline & & & Ball carries & $4.0(4.0)$ & NR \\
\hline & & & Tackles and ball carries & $21.0(12.0)$ & $0.30(0.30)$ \\
\hline & & Outside backs & Tackles & $8.0(10.0)$ & NR \\
\hline & & & Ball carries & $9.0(4.0)$ & NR \\
\hline & & & Tackles and ball carries & $19.0(9.0)$ & $0.30(0.10)$ \\
\hline Gabbett \& Ryan [67] & NRL (2008-2009) & Team & Tackles & $24.0(\mathrm{NR})$ & $N R$ \\
\hline \multirow[t]{12}{*}{ Gabbett et al. [44] } & NRL (2008-2010) & Hit-up forwards & Tackles (total defensive) & $23.0(21.0,25.0)^{a}$ & $N R$ \\
\hline & & & Ball carries (total attack) & $13.0(11.0,15.0)^{\mathrm{a}}$ & NR \\
\hline & & & Tackles and ball carries & $36.0(32.0,40.0)^{\mathrm{a}}$ & NR \\
\hline & & Wide-running forwards & Tackles (total defensive) & $30.0(26.0,34.0)^{\mathrm{a}}$ & NR \\
\hline & & & Ball carries (total attack) & $17.0(13.0,21.0)^{a}$ & NR \\
\hline & & & Tackles and ball carries & $47.0(42.0,52.0)^{\mathrm{a}}$ & NR \\
\hline & & Adjustables & Tackles (total defensive) & $19.0(15.0,23.0)^{a}$ & $N R$ \\
\hline & & & Ball carries (total attack) & $10.0(7.0,13.0)^{\mathrm{a}}$ & NR \\
\hline & & & Tackles and ball carries & $29.0(26.0,32.0)^{a}$ & $N R$ \\
\hline & & Outside backs & Tackles & $11.0(9.0,13.0)^{\mathrm{a}}$ & NR \\
\hline & & & Ball carries & $13.0(12.0,14.0)^{a}$ & NR \\
\hline & & & Tackles and ball carries & $24.0(22.0,27.0)^{\mathrm{a}}$ & $N R$ \\
\hline \multirow[t]{2}{*}{ Gabbett et al. [49] } & NRL (2008-2011) & Team & Tackles & $17.1(9.1)$ & $N R$ \\
\hline & & & Ball carries & $8.8(2.8)$ & NR \\
\hline
\end{tabular}


Table 3 Characteristics of collisions during match-play recorded by video notational analysis (Continued)

\begin{tabular}{|c|c|c|c|c|c|}
\hline Study & Competition (season[s]) & Positional Group & Type of collision recorded & $\begin{array}{l}\text { Frequency of } \\
\text { collisions }(n=) \\
\text { mean }( \pm S D)\end{array}$ & $\begin{array}{l}\text { Relative frequency } \\
\text { of collisions }\left(n \cdot \mathrm{min}^{-1}=\right) \\
\text { mean }( \pm \text { SD) }\end{array}$ \\
\hline \multirow[t]{22}{*}{ King et al. [41] } & \multirow[t]{11}{*}{ International (2008) } & Game & Tackles and ball carries & 620.6 (NR) & NR \\
\hline & & \multirow[t]{2}{*}{ Backs } & Tackles & $377.0(22.9)$ & NR \\
\hline & & & Ball carries & 285.7 (21.6) & NR \\
\hline & & \multirow[t]{2}{*}{ Forwards } & Tackles & $623.0(29.4)$ & NR \\
\hline & & & Ball carries & $238.1(19.6)$ & NR \\
\hline & & \multirow[t]{2}{*}{ Hit-up forwards } & Tackles & 386.5 (23.3) & NR \\
\hline & & & Ball carries & $340.9(32.4)$ & NR \\
\hline & & \multirow[t]{2}{*}{ Adjustables } & Tackles & $404.8(23.8)$ & NR \\
\hline & & & Ball carries & $224.3(26.2)$ & NR \\
\hline & & \multirow[t]{2}{*}{ Outside backs } & Tackles & $208.6(17.1)$ & NR \\
\hline & & & Ball carries & 434.9 (36.6) & NR \\
\hline & \multirow[t]{11}{*}{ NRL (2008) } & Game & Tackles and ball carries & 650.8 (NR) & NR \\
\hline & & \multirow[t]{2}{*}{ Backs } & Tackles & $343.6(22.5)$ & NR \\
\hline & & & Ball carries & $257.2(19.8)$ & NR \\
\hline & & \multirow[t]{2}{*}{ Forwards } & Tackles & $656.4(31.2)$ & NR \\
\hline & & & Ball carries & $229.7(18.7)$ & NR \\
\hline & & \multirow[t]{2}{*}{ Hit-up forwards } & Tackles & 378.5 (23.6) & NR \\
\hline & & & Ball carries & $366.3(34.1)$ & NR \\
\hline & & \multirow[t]{2}{*}{ Adjustables } & Tackles & $451.0(25.7)$ & NR \\
\hline & & & Ball carries & $199.1(12.9)$ & NR \\
\hline & & \multirow[t]{2}{*}{ Outside backs } & Tackles & $170.5(15.9)$ & NR \\
\hline & & & Ball carries & $434.6(37.0)$ & NR \\
\hline \multirow[t]{3}{*}{ King et al. [28] } & \multirow[t]{3}{*}{ NRL (NR) } & Game & Tackles (completed) & $590.0(50.0)$ & $N R$ \\
\hline & & Backs & $\begin{array}{l}\text { Tackles and ball carries } \\
\text { (completed and missed) }\end{array}$ & $14.6(7.7)$ & NR \\
\hline & & Forwards & $\begin{array}{l}\text { Tackles and ball carries } \\
\text { (completed and missed) }\end{array}$ & $27.1(8.3)$ & $N R$ \\
\hline \multirow[t]{6}{*}{ McLellan \& Lovell [56] } & \multirow[t]{6}{*}{$N R L(N R)$} & \multirow[t]{2}{*}{ Team } & Tackles & $19.9(10.5)$ & $N R$ \\
\hline & & & Ball carries & $12.2(3.6)$ & NR \\
\hline & & \multirow[t]{2}{*}{ Backs } & Tackles & $10.7(8.9)$ & NR \\
\hline & & & Ball carries & $11.7(4.6)$ & NR \\
\hline & & \multirow[t]{2}{*}{ Forwards } & Tackles & $26.1(15.3)$ & NR \\
\hline & & & Ball carries & $13.8(5.2)$ & NR \\
\hline \multirow[t]{6}{*}{ McLellan et al. [38] } & \multirow[t]{6}{*}{ NRL (NR) } & \multirow[t]{2}{*}{ Team } & Tackles & $14.9(10.5)$ & NR \\
\hline & & & Ball carries & $10.2(3.8)$ & NR \\
\hline & & \multirow[t]{2}{*}{ Backs } & Tackles & $10.7(8.0)$ & NR \\
\hline & & & Ball carries & $9.7(3.5)$ & NR \\
\hline & & \multirow[t]{2}{*}{ Forwards } & Tackles & $20.1(11.3)$ & NR \\
\hline & & & Ball carries & $10.9(4.2)$ & NR \\
\hline Sirotic et al. [32] & NRL (2004-2005) & Team & Tackles & NR & $0.25(0.16)$ \\
\hline & & & Ball carries & NR & $0.15(0.08)$ \\
\hline & NSWCup (2004-2005) & Team & Tackles & NR & $0.28(0.16)$ \\
\hline & & & Ball carries & NR & $0.15(0.08)$ \\
\hline
\end{tabular}


Table 3 Characteristics of collisions during match-play recorded by video notational analysis (Continued)

\begin{tabular}{|c|c|c|c|c|c|}
\hline Study & Competition (season[s]) & Positional Group & Type of collision recorded & $\begin{array}{l}\text { Frequency of } \\
\text { collisions }(n=) \\
\text { mean }( \pm \text { SD) }\end{array}$ & $\begin{array}{l}\text { Relative frequency } \\
\text { of collisions }\left(n \cdot \mathrm{min}^{-1}=\right. \\
\text { mean }( \pm \text { SD) }\end{array}$ \\
\hline \multirow[t]{4}{*}{ Sirotic et al. [59] } & \multirow[t]{4}{*}{ NRL (2004-2005) } & \multirow[t]{2}{*}{ Backs } & Tackles & $N R$ & $0.12(0.09)$ \\
\hline & & & Ball carries & $N R$ & $0.11(0.04)$ \\
\hline & & \multirow[t]{2}{*}{ Forwards } & Tackles & NR & $0.41(0.07)$ \\
\hline & & & Ball carries & $N R$ & $0.25(0.09)$ \\
\hline \multirow[t]{3}{*}{ Sperenza et al. [61] } & \multirow[t]{3}{*}{ QCup (2014) } & Team & Tackles & 18.0 (NR) & NR \\
\hline & & Backs & Tackles & $13.2(8.5)$ & $N R$ \\
\hline & & Forwards & Tackles & $24.3(6.5)$ & NR \\
\hline \multirow[t]{6}{*}{ Twist et al. [62] } & \multirow[t]{6}{*}{ SL (2010) } & \multirow[t]{3}{*}{ Backs } & Tackles & $13.6(7.9)$ & $0.20(0.10)$ \\
\hline & & & Ball carries & $11.6(3.4)$ & $0.10(0.04)$ \\
\hline & & & Tackles and ball carries & $25.2(8.0)$ & $0.30(0.10)$ \\
\hline & & \multirow[t]{3}{*}{ Forwards } & Tackles & $25.5(13.7)$ & $0.50(0.20)$ \\
\hline & & & Ball carries & $12.7(6.1)$ & $0.30(0.10)$ \\
\hline & & & Tackles and ball carries & $38.2(18.7)$ & $0.70(0.30)$ \\
\hline \multirow[t]{4}{*}{ Woods et al. [1] } & \multirow[t]{2}{*}{ NRL (2016) } & \multirow[t]{2}{*}{ Game } & Tackles & $314.3(15.9)$ & NR \\
\hline & & & Ball carries & $164.3(13.5)$ & NR \\
\hline & \multirow[t]{2}{*}{ SL (2016) } & \multirow[t]{2}{*}{ Game } & Tackles & $336.1(11.8)$ & NR \\
\hline & & & Ball carries & $179.0(8.1)$ & NR \\
\hline \multirow[t]{4}{*}{ Woods et al. [64] } & \multirow[t]{2}{*}{ NRL (2016) } & \multirow[t]{2}{*}{ Game } & Tackles & $325.0(39.7)$ & NR \\
\hline & & & Ball carries & $170.2(19.8)$ & NR \\
\hline & \multirow[t]{2}{*}{ NYC (2016) } & \multirow[t]{2}{*}{ Game } & Tackles & $283.4(35.6)$ & NR \\
\hline & & & Ball carries & $147.2(17.4)$ & NR \\
\hline
\end{tabular}

Data are reported as mean \pm Standard Deviation (SD) unless otherwise stated. ${ }^{\text {a Data }}$ are reported as mean ( $\pm 95 \%$ confidence intervals) as SD was not able to be determined due to insufficient information. Game = results for both teams involved at the game level, NR Not reported, NRL National Rugby League, NYC National Youth Competition, NSWCup New South Wales Cup, QCup Queensland Cup, SL Super League, Team = results at the individual team level

26.0 collisions per match $[24,47,50]$ (see Table 4$)$. When examined at the positional group level, adjustables completed 16.4 to 34.0 collisions per match, whilst studies showed outside backs complete between 14.8 and 28.0 collisions per match $[24,25,31,47]$. Two studies reported collisions per match for hit-up forwards and wide-running forwards based on microtechnology, with hit-up and wide-running forwards undertaking 37.0 to 42.0 and 28.0 to 45.0 collisions per match, respectively [25, 31].

For relative collision frequency as reported via microtechnology, forwards completed between 0.35 and $0.77 n \cdot \mathrm{min}^{-1}$ of match-play $[24,47,50]$, which was greater than the one study which reported the relative frequency of backs $\left(n \cdot \min ^{-1}=0.30\right)$ [50]. These differences were mirrored when relative collisions were examined at the positional subgroup level, with hit-up forwards undertaking greater collisions per minute $\left(n \cdot \mathrm{min}^{-1}=0.69-1.09\right)$ than adjustables $\left(n \cdot \mathrm{min}^{-1}=0.23-0.79\right)$ and outside backs $\left(n \cdot \mathrm{min}^{-1}=\right.$ 0.19-0.48) (see Table 4) [24, 25, 31, 47, 48, 52]. Two studies investigated the relative frequency of collisions completed by wide-running forwards as reported via microtechnology, identifying that wide-running forwards undertook 0.59 to 0.76 collisions per min of match-play $[25,31]$.

\section{Collision intensity Video notational analysis}

Three studies reported metrics associated with the intensity of collisions as subjectively derived from video analysis [31, 33, 67]. One study concluded that both professional and semi-professional athletes undertake a similar velocity into contact ( 2.81 vs. $2.76 \mathrm{~m} \cdot \mathrm{sec}^{-1}$ ) [67]. Furthermore, the influence of progressive fatigue on acceleration into contact has been examined through frame by frame analysis of video, with a gradual decrease in acceleration reported with increased levels of fatigue [33]. Acceleration into contact decreased from $3.8 \mathrm{~m} \cdot \mathrm{sec}^{-2}$ with low fatigue, to 2.3 $\mathrm{m} \cdot \mathrm{sec}^{-2}$ during moderate fatigue, and to $1.7 \mathrm{~m} \cdot \mathrm{sec}^{-2}$ during periods of heavy fatigue.

Finally, one study investigated collision intensity by characterising each collision through a mild, moderate, or heavy rating system [31]. In this system, a mild collision occurred when a player made contact with a player 


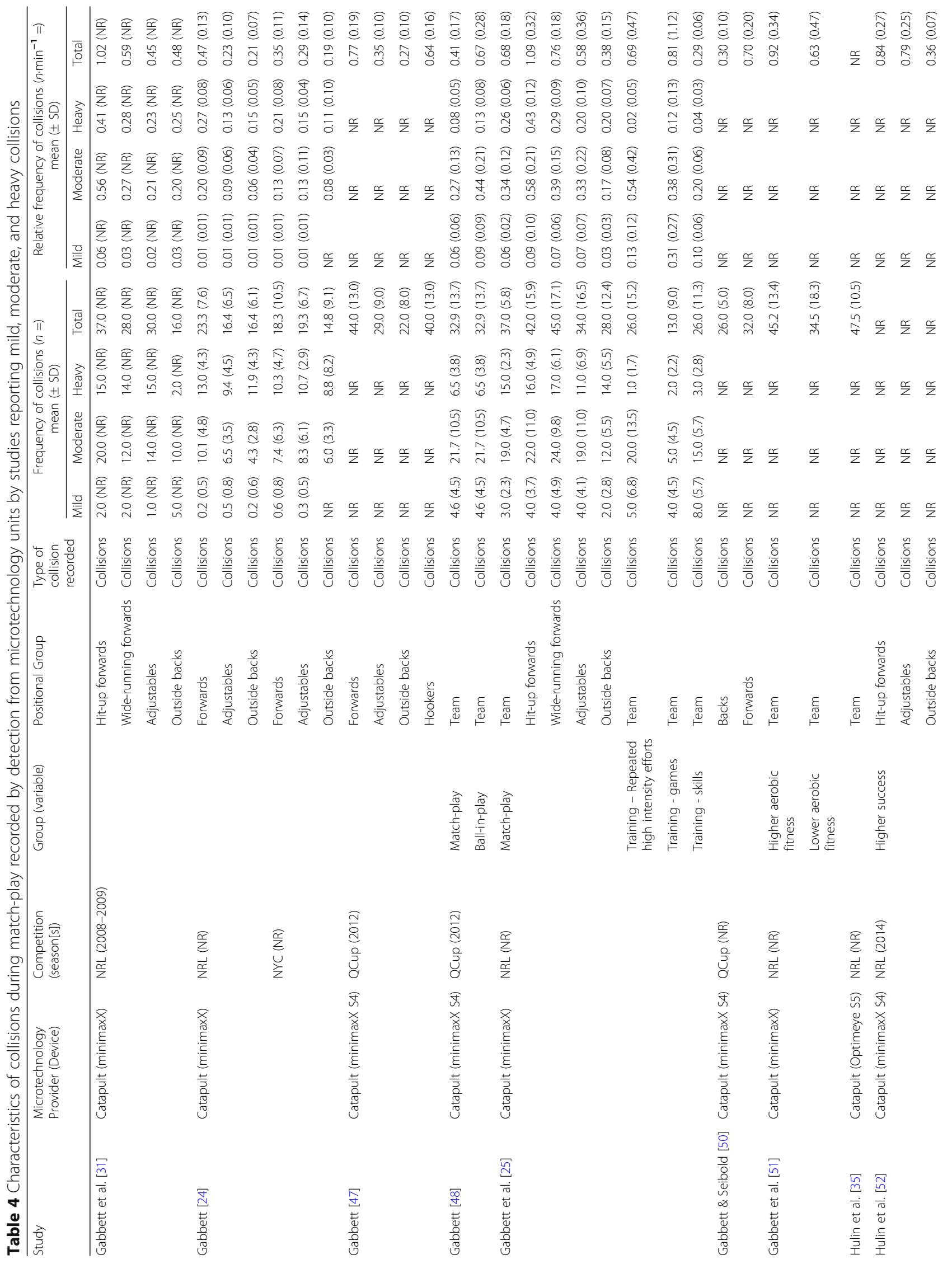




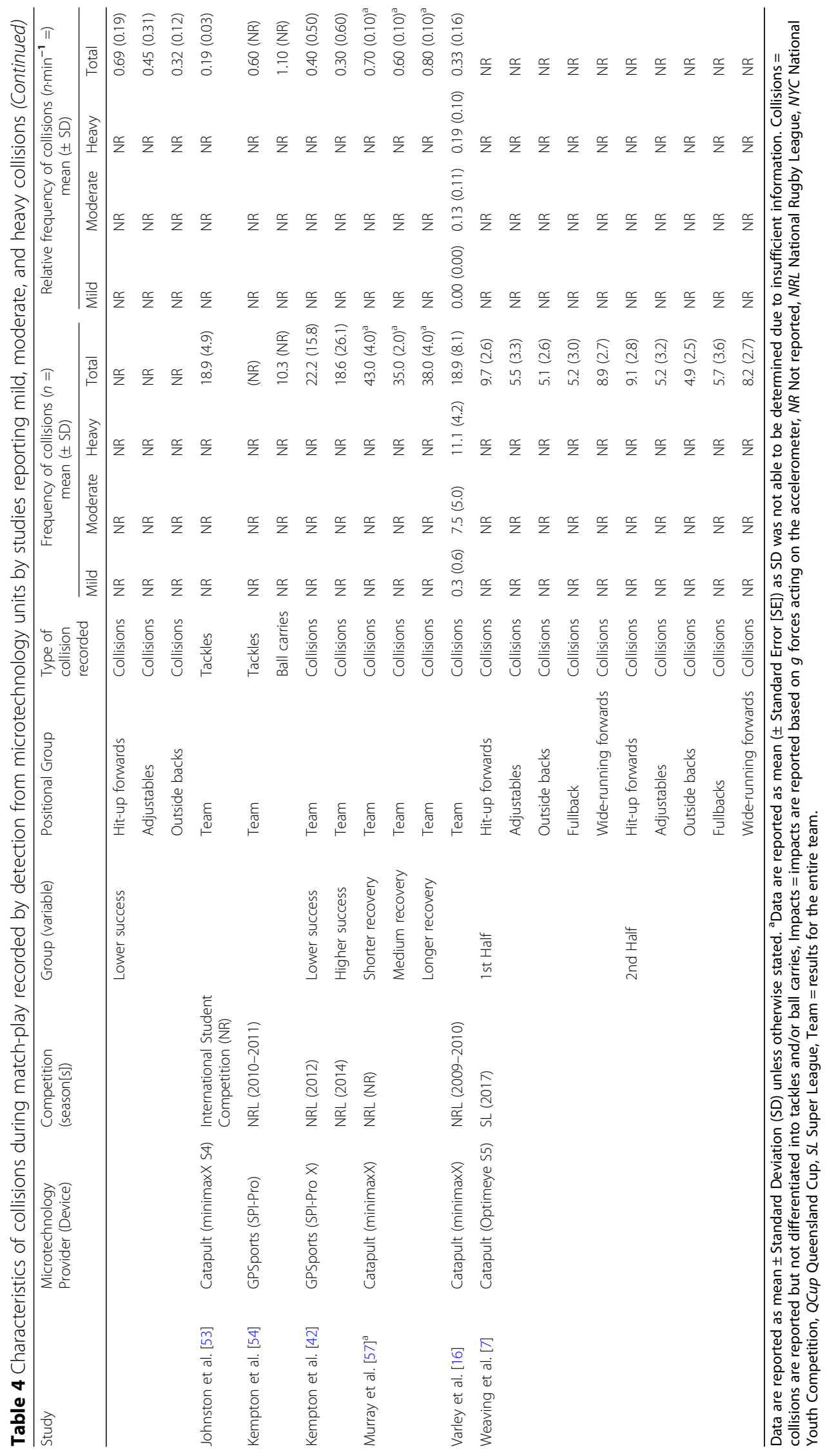


Table 5 Zone characteristics for microtechnology devices using specific $g$ force zones

\begin{tabular}{llllllll}
\hline Device & Studies & Zone 1 & Zone 2 & Zone 3 & Zone 4 & Zone 5 & Zone 6 \\
\hline SPI-Pro X & Cummins \& Orr [60] & $<5.9 \mathrm{~g}$ & $6.0-6.9 \mathrm{~g}$ & $7.0-7.9 \mathrm{~g}$ & $8.0-9.9 \mathrm{~g}$ & $10.0-11.9 \mathrm{~g}$ & $>12.0 \mathrm{~g}$ \\
SPI-Pro XII & Cummins \& Orr [26] & $<5.9 \mathrm{~g}$ & $6.0-6.9 \mathrm{~g}$ & $7.0-7.9 \mathrm{~g}$ & $8.0-9.9 \mathrm{~g}$ & $10.0-11.9 \mathrm{~g}$ & $>12.0 \mathrm{~g}$ \\
SPI-Pro XII & Evans et al. [65] & $7-9 \mathrm{~g}$ & $9-11 \mathrm{~g}$ & $11-13 \mathrm{~g}$ & $13-15 \mathrm{~g}$ & $\mathrm{NR}$ & $\mathrm{NR}$ \\
SPI-Pro & Lovell et al. [55]; McLellan \& Lovell [56]; & $5-6 \mathrm{~g}$ & $6.01-6.5 \mathrm{~g}$ & $6.51-7.0 \mathrm{~g}$ & $7.01-8.0 \mathrm{~g}$ & $8.01-10.0 \mathrm{~g}$ & $>10.0 \mathrm{~g}$ \\
& McLellan et al. [38]; Weaving et al. [63]. & & & & & \\
MinimaxX S4 & Oxendale et al. [58]. & $2-3 \mathrm{~g}$ & $3-4.5 \mathrm{~g}$ & $4.5-6 \mathrm{~g}$ & $6-8 \mathrm{~g}$ & $>8 \mathrm{~g}$ & $\mathrm{NR}$ \\
\hline
\end{tabular}

NR Not reported

but was able to continue forward progress, and a moderate collision was coded when an athlete made contact and momentum continued until finally being tackled. Lastly, a heavy collision was coded when momentum was halted and the athlete forced backwards [31]. Of the 237 collisions analysed using this system, 24 were considered mild, 46 were considered moderate, and 119 were considered heavy. This represented a $63 \%$ difference between mild and moderate collisions, and a $133 \%$ difference between mild and heavy coded collisions [31].

\section{Microtechnology}

Pooled analysis of microtechnology-based studies identified 3.2 mild, 17.0 moderate and 7.9 heavy collisions per match (see Fig. 6a-c).

At the positional group level, hit-up $(n=2.0-4.0)$ and wide-running forwards $(n=2.0-4.0)$ were associated with the greatest number of mild collisions per match when compared to outside backs $(n=0.2-5.0)$ and adjustables $(n=0.3-4.0)[24,25,31]$. These positional group differences were mirrored within moderate collisions with wide-running ( $n=12.0-24.0)$ and hit-up forwards $(n=$ 20.0-22.0) completing more moderate collisions than adjustables $(n=6.5-19.0)$ and outside backs $(n=4.3-12.0)$ $[24,25,31]$. Finally, hit-up $(n=15.0-16.0)$ and widerunning forwards $(n=14.0-17.0)$ completed a greater frequency of heavy collisions per match than outside backs $(n=2.0-14.0)$ and adjustables $(n=9.4-15.0)[24,25,31]$.

Eight of the included studies [26, 38, 55, 56, 58, 60, 63, 65] reported collision intensity based on accelerometer load ( $g$ forces) which was divided into discrete intensity zones (see Table 5). These studies reported on four unique intensity zones ranging from 2-3 to $>12 g$ zone configurations from microtechnology devices manufactured by GPSports (SPIPro, SPI-Pro X, SPI-Pro XII devices), and Catapult (mini$\operatorname{maxX}$ S4 device) (see Table 5). A number of these studies $[38,55,56,63,65]$ reported collisions using a manufacturer derived impact metric (see Table 6). Given the disparity between reporting impacts and collisions, and in the zone classification systems utilised in these studies, further comparisons were not able to be performed as the methods and data were heterogeneous.

\section{Discussion}

To our knowledge, this is the first systematic review, including a meta-analysis to specifically synthesise the current methods of analysing the frequency and intensity of collisions in rugby league. The review demonstrates that video-based notational analysis and microtechnology devices are the two primary methods utilised to examine the frequency and intensity of collisions in rugby league. Collectively, forwards experience a greater dose of collisions than backs which is primarily attributable to a greater tackle frequency. Overall, collisions have been quantified using a variety of data collection methods with a lack of consistency in regards to positional groups as well as intensity descriptors.

\section{Collision frequency}

From a video analysis perspective, there are disparities in the collision demands across positional groups, with forwards completing a greater number of collisions per match when compared to backs. This disparity is primarily associated with a greater tackle frequency for forwards, with ball carry frequency being similar across the positional groups. Forwards exhibit a near three-fold increase in the number of tackles per minute of match-play when compared to backs, with the relative number of ball carries also being higher for forwards. This pattern likely reflects the tactical demands of modern rugby league, with teams utilising interchange players to complete tackles and ball carries during their time on field. This translates to a greater defensive workload for these players who occupy the middle of the defensive line (i.e. the forward positional groups), resulting in a greater number of collisions completed relative to the players time on field.

These findings were also reflected in the absolute and relative collision frequencies as quantified via microtechnology. Forwards and their positional subgroups of hit-up and wide-running forwards complete a greater number of collisions in absolute terms and relative to their time on the field of play. High heterogeneity was observed in the collision demands derived from microtechnology at the team level. This may be due to the majority of studies focusing on a single team or cohort, and the unique physical and tactical aspects placed upon those players not generalising across teams or cohorts. Alternatively, the high 


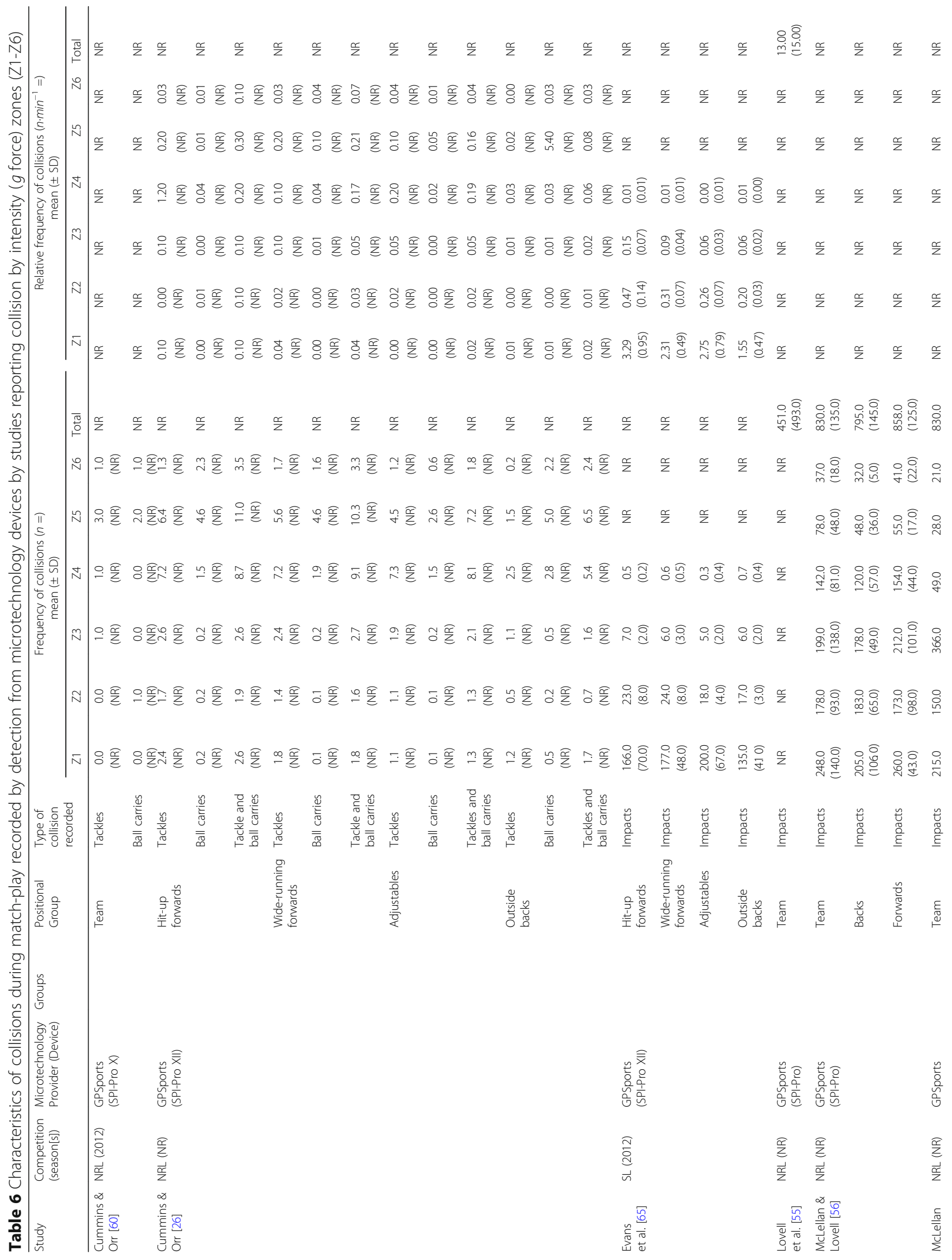




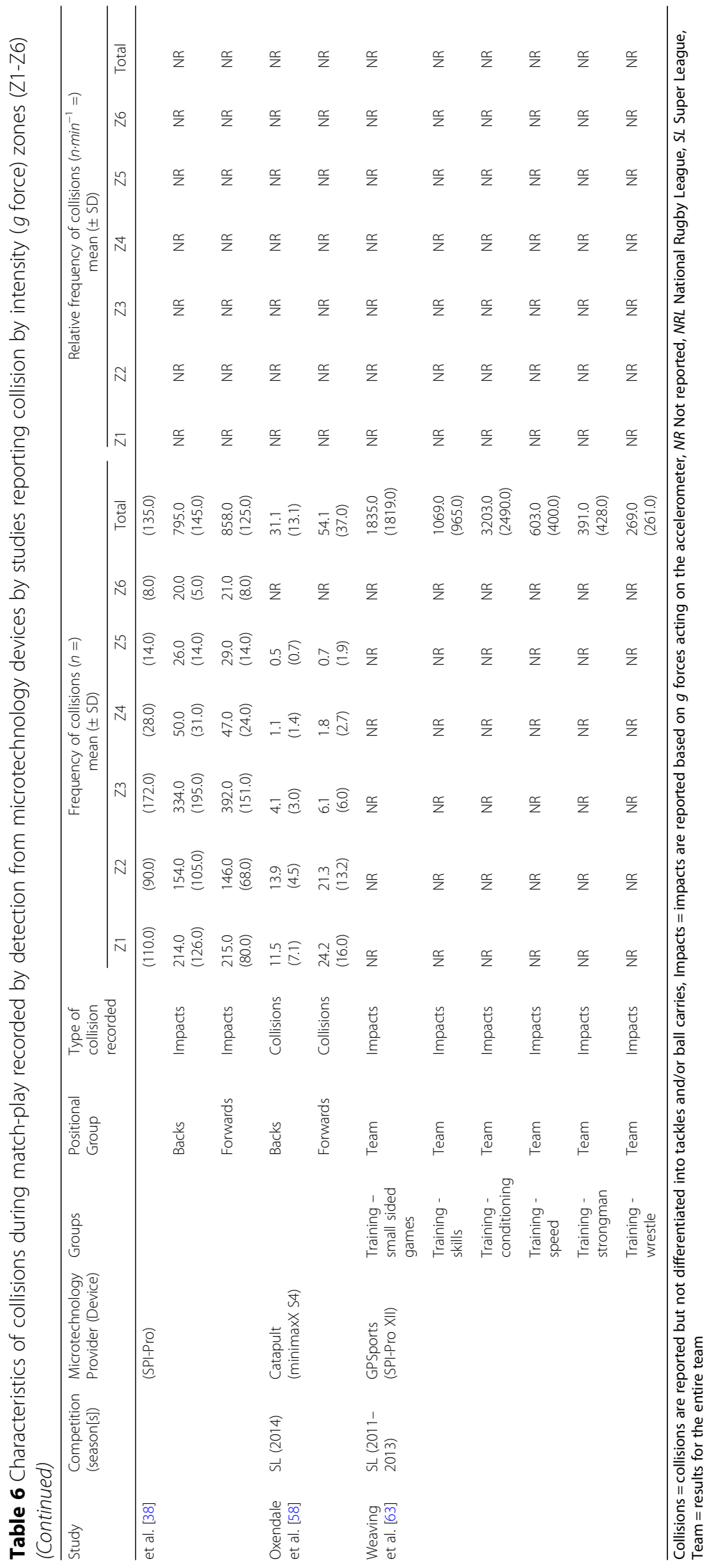




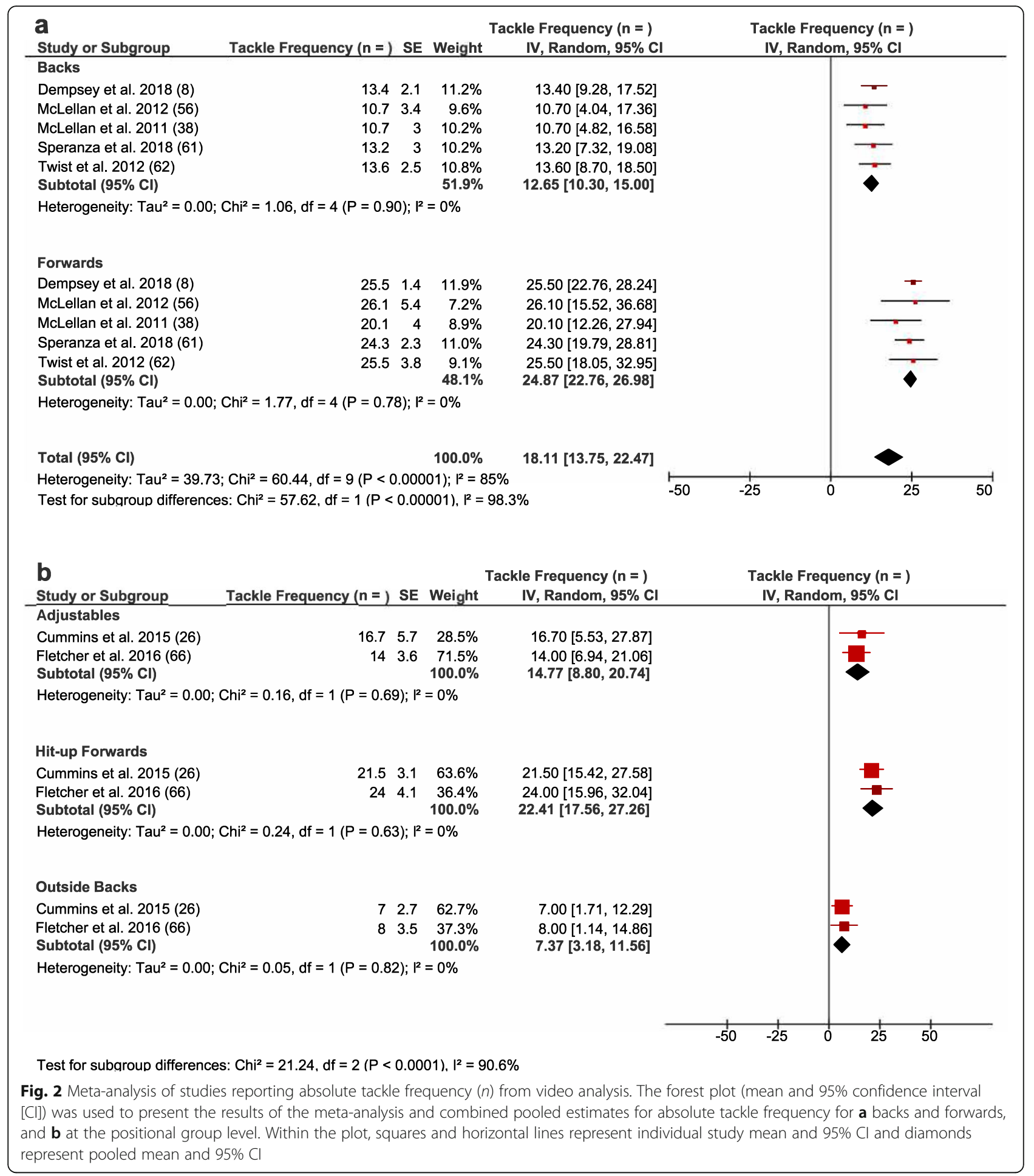

heterogeneity might be a function of the different microtechnology devices utilised between studies. Further granular analysis of differences in the type of collisions is presently not possible, as microtechnology does not permit the differentiation of collisions into tackles and ball carries.
The lack of consensus regarding the definition of tackles and collisions is a potential issue within the current literature. Specifically, a number of video-based studies used the definition described by Gissane et al. [68] wherein a tackle is defined as when "... the ball carrier is held by one or more of the 


\begin{tabular}{|c|c|c|c|c|c|c|c|}
\hline \multirow{2}{*}{$\begin{array}{l}\text { a } \\
\text { Study or Subgroup } \\
\text { Backs }\end{array}$} & Ball Carry Frequency $(n=)$ SE & Weight & \multirow[t]{2}{*}{$\begin{array}{l}\text { Ball Carry Frequency }(\mathrm{n}=\text { ) } \\
\text { IV, Random, } 95 \% \mathrm{Cl}\end{array}$} & \multicolumn{4}{|c|}{$\begin{array}{l}\text { Ball Carry Frequency }(n=) \\
\text { IV, Random, } 95 \% \mathrm{Cl}\end{array}$} \\
\hline & & & & & & & \\
\hline Dempsey et al. $2018(8)$ & 11.91 .2 & $11.0 \%$ & $11.90[9.55,14.25]$ & & & $\mp$ & \\
\hline McLellan et al. 2012 (56) & $11.7 \quad 1.7$ & $5.5 \%$ & $11.70[8.37,15.03]$ & & & $\longrightarrow$ & \\
\hline McLellan et al. 2011 (38) & $9.7 \quad 1.3$ & $9.3 \%$ & $9.70[7.15,12.25]$ & & & - & \\
\hline Twist et al. 2012 (62) & $\begin{array}{lll}11.6 & 1.1\end{array}$ & $13.0 \%$ & $11.60[9.44,13.76]$ & & & 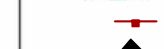 & \\
\hline Subtotal $(95 \% \mathrm{Cl})$ & 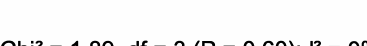 & $38.8 \%$ & $11.24[9.99,12.49]$ & & & $\boldsymbol{\nabla}$ & \\
\hline \multicolumn{8}{|c|}{ Heterogeneity: $\operatorname{Tau}^{2}=0.00 ; \mathrm{Chi}^{2}=1.89, \mathrm{df}=3(\mathrm{P}=0.60) ; \mathrm{I}^{2}=0 \%$} \\
\hline \multicolumn{8}{|l|}{ Forwards } \\
\hline Dempsey et al. 2018 (8) & 10.50 .6 & $43.8 \%$ & $10.50[9.32,11.68]$ & & & 를 & \\
\hline McLellan et al. $2012(56)$ & 13.81 .8 & $4.9 \%$ & $13.80[10.27,17.33]$ & & & $\longrightarrow$ & \\
\hline McLellan et al. 2011 (38) & 10.91 .5 & $7.0 \%$ & $10.90[7.96,13.84]$ & & & $\longrightarrow$ & \\
\hline Twist et al. 2012 (62) & $\begin{array}{lll}12.7 & 1.7\end{array}$ & $5.5 \%$ & $12.70[9.37,16.03]$ & & & $\overrightarrow{\mathbf{n}}$ & \\
\hline \multicolumn{7}{|c|}{ Heterogeneity: $\mathrm{Tau}^{2}=0.63 ; \mathrm{Chi}^{2}=4.12, \mathrm{df}=3(P=0.25) ; 1^{2}=27 \%$} & \\
\hline Total $(95 \% \mathrm{Cl})$ & & $100.0 \%$ & $11.10[10.32,11.88]$ & & & $\bullet$ & \\
\hline \multicolumn{4}{|c|}{$\begin{array}{l}\text { Heterogeneity: } \mathrm{Tau}^{2}=0.00 ; \mathrm{Ch}^{2}=6.09, \mathrm{df}=7(P=0.53) ; \mathrm{l}^{2}=0 \% \\
\text { Test for subaroup differences: } \mathrm{Ch}^{2}=0.01, \mathrm{df}=1(P=0.91), \mathrm{I}^{2}=0 \%\end{array}$} & -20 & -10 & 10 & 20 \\
\hline b & & & Ball Carry Frequency $(n=)$ & \multirow{2}{*}{\multicolumn{4}{|c|}{$\begin{array}{l}\text { Ball Carry Frequency }(n=) \\
\text { IV, Random, } 95 \% \mathrm{Cl}\end{array}$}} \\
\hline Study or Subgroup & Ball Carry Frequency $(n=)$ SE & Weight & IV, Random, $95 \% \mathrm{Cl}$ & & & & \\
\hline \multicolumn{8}{|l|}{ Adjustables } \\
\hline Cummins et al. 2015 (26) & 4.92 .1 & $24.6 \%$ & $4.90[0.78,9.02]$ & & & & \\
\hline Fletcher et al. $2016(66)$ & 41.2 & $75.4 \%$ & $4.00[1.65,6.35]$ & & & & \\
\hline Subtotal $(95 \% \mathrm{Cl})$ & & $100.0 \%$ & $4.22[2.18,6.26]$ & & & & \\
\hline \multicolumn{8}{|c|}{ Heterogeneity: $\mathrm{Tau}^{2}=0.00 ; \mathrm{Chi}^{2}=0.14, \mathrm{df}=1(P=0.71) ; \mathrm{I}^{2}=0 \%$} \\
\hline \multicolumn{8}{|l|}{ Hit-up Forwards } \\
\hline Cummins et al. 2015 (26) & 8.91 .9 & $41.5 \%$ & $8.90[5.18,12.62]$ & & & - & \\
\hline Fletcher et al. 2016 (66) & 8.51 .6 & $58.5 \%$ & $8.50[5.36,11.64]$ & & & & \\
\hline \multicolumn{8}{|c|}{ Heterogeneity: Tau $^{2}=0.00 ; \mathrm{Chi}^{2}=0.03, \mathrm{df}=1(P=0.87) ; \mathrm{I}^{2}=0 \%$} \\
\hline \multicolumn{8}{|l|}{ Outside Backs } \\
\hline Cummins et al. 2015 (26) & 11.20 .9 & $61.9 \%$ & $11.20[9.44,12.96]$ & & & $\mathrm{E}$ & \\
\hline Fletcher et al. 2016 (66) & $\begin{array}{ll}9 & 1.4\end{array}$ & $38.1 \%$ & $9.00[6.26,11.74]$ & & & & \\
\hline Subtotal $(95 \% \mathrm{Cl})$ & & $100.0 \%$ & $10.36[8.27,12.46]$ & & & & \\
\hline \multicolumn{8}{|c|}{ Heterogeneity: $\mathrm{Tau}^{2}=1.03 ; \mathrm{Chi}^{2}=1.75, \mathrm{df}=1(\mathrm{P}=0.19) ; \mathrm{I}^{2}=43 \%$} \\
\hline & & & & -20 & -10 & 10 & 20 \\
\hline \multicolumn{8}{|c|}{ Test for subgroup differences: $\mathrm{Chi}^{2}=17.95, \mathrm{df}=2(\mathrm{P}=0.0001), \mathrm{I}^{2}=88.9 \%$} \\
\hline \multicolumn{8}{|c|}{$\begin{array}{l}\text { Fig. } 3 \text { Meta-analysis of studies reporting ball carry frequency }(n) \text { from video analysis. This forest plot (mean and } 95 \% \text { confidence interval }[\mathrm{Cl}] \text { ) was } \\
\text { used to present the results of the meta-analysis and combined pooled estimates for absolute ball carry frequency for a backs and forwards, and } \\
\text { b at the positional group level. Within the plot, squares and horizontal lines represent individual study mean and } 95 \% \mathrm{Cl} \text { and diamonds represent } \\
\text { pooled mean and } 95 \% \mathrm{Cl}\end{array}$} \\
\hline
\end{tabular}

opposing players and either the ball or hand of the arm holding the ball makes contact with the ground or the ball carriers cannot make further progress." [28, 41, 44]. Other studies focusing on microtechnology-based collisions however, have defined the collision as occurring when a player makes contact with another player or the ground, which results in an alteration to the player's momentum or direction of travel [31, 35]. The differences in definitions of tackles and collisions have the potential to alter the frequency in which these actions are counted and hinders the ability to translate findings into practical recommendations.

\section{Collision intensity}

Compared to literature quantifying the absolute and relative collision frequency demands of match-play, there is a paucity of research on the intensity of collisions within rugby league. Further limiting such research is the lack of consistency in the quantification of 


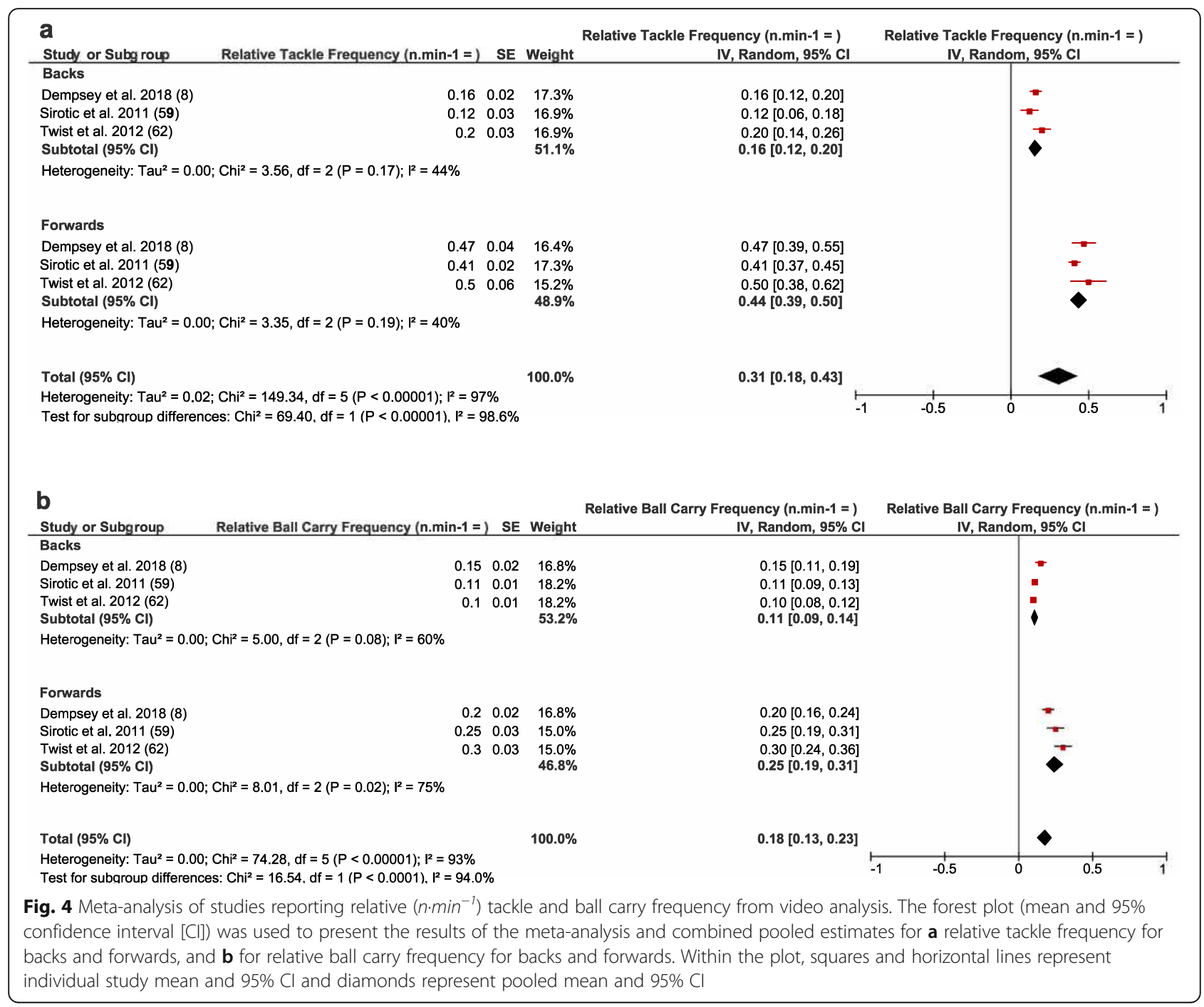

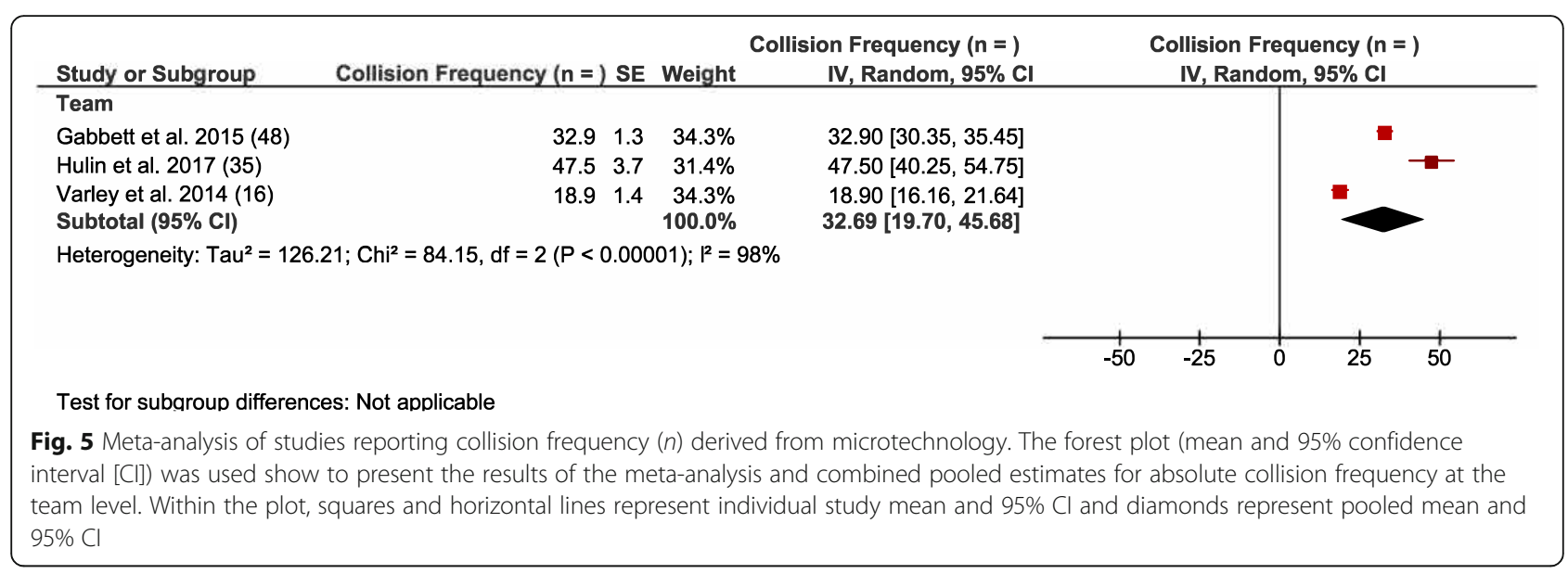




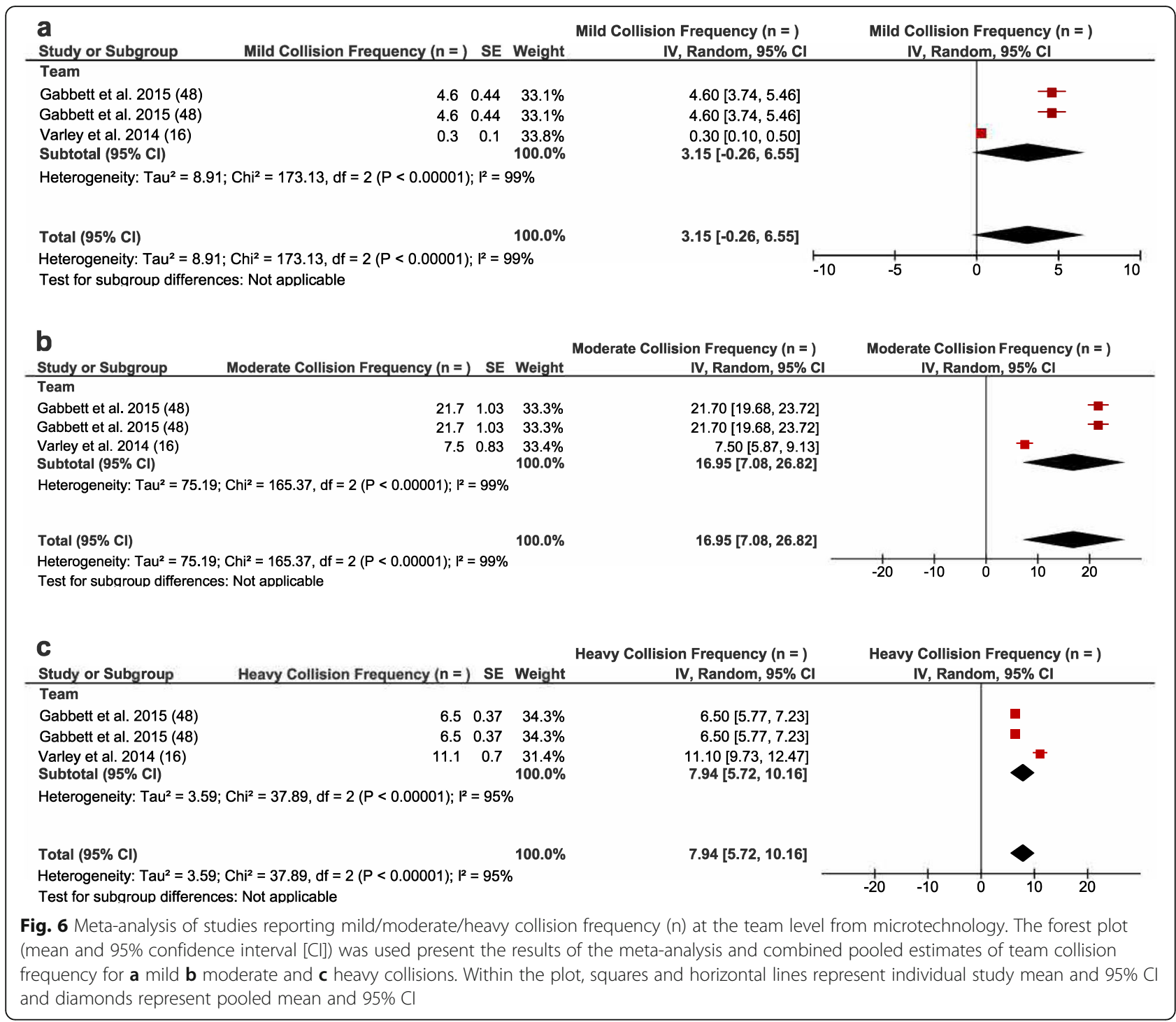

collision intensity between studies. Through video analysis, studies have attempted to quantify collision intensity by calculating the velocity and accelerations into contact $[67,69]$. This process involves manually coding video frame-by-frame before the athlete enters a collision to derive velocity and acceleration and as such is time-consuming [69], and may be influenced by the subjective nature of video analysis. The time taken to code each frame and the manual nature of this process means that this information cannot be used in real time or close to real time to influence decision making. Using velocity into contact as an intensity metric makes a number of assumptions that primarily relate to velocity equating to impact forces, and in turn into dominance in the collision. This relationship has been examined in rugby union, whereby velocity into contact, but not impact force, was greater for dominant tackles and carries when compared to neutral and passive tackles and ball carries [70]. Gabbett and Ryan investigated the differences between professional and semi-professional rugby league athletes in regards to velocity into contact and subjective ratings of tackle performance [67]. Despite large differences in tackle performance between competition standards, minimal differences were noted for velocity into contact, suggesting that collision dominance may not be related to the velocity that a player approaches the collision. To our knowledge, this is the only study to investigate velocities and collision dominance in rugby league, and further research in this area is warranted.

Literature examining collision intensity via microtechnology has also been limited due to methodological inconsistencies with accelerometer-derived classification zones used to categorise impacts from low through to high intensity [56]. In theory, this process provides practitioners with an understanding of the accelerometer load that includes the 
accumulation of the tri-axial forces acting upon the accelerometer from actions such as change of direction movements, accelerations and decelerations, and collisions [55]. As such, an impact metric provides limited information for the practitioner in regards to collision intensity and instead is indicative of a 'global' accelerometer load [4]. Furthermore, intensity-based research has employed a number of different devices with four to six different intensity zone categories ranging from 2-3 $g$ to $>12 g$ (see Table 5), and estimated collision intensity via qualitatively grouping collisions into categories of mild, moderate, or heavy. Given the lack of methodological consistency within and between studies utilising $g$ force-based intensity zones, further comparison and analysis of these findings is difficult. As such, as previously highlighted by Cummins et al. [15], a consensus on the definition of zone intensities and descriptors for both impact metrics and accelerometer loads is required in order to facilitate comparisons within individual sporting codes and levels of participation. Such consistency could provide insights which are generalisable between teams and positional groups, enabling meaningful analyses, and ensuring athletes are exposed to appropriate collision loads, which is critical to both injury-prevention and physical conditioning.

Beyond the initial velocity and contact force, another factor that may influence the intensity of the collision is the post-contact wrestle phase of the tackle or ball carry. During this phase the athletes involved wrestle or grapple to achieve dominance in the tackle and this necessitates large muscular force generation whilst in a near stationary position [71]. These static exertions involve isometric and eccentric muscle actions that are likely to produce extensive muscle damage [27], and incur substantial energetic costs [71]. Despite the apparent high energetic demand of such events, microtechnology is incapable of quantifying the work completed in a stationary position due to minimal displacement of the device [71, 72]. As such, microtechnology devices are unable to account for the physiological demand that occurs during static exertions, and are therefore, not an appropriate tool to monitor collision intensity in the post-contact wrestle phase.

\section{Microtechnology validity}

There has been an increase in the number of studies examining and reporting the collision demands of rugby league training and match-play. The use of microtechnology to automatically quantify the frequency of collision events appears to be an emerging area of research. Despite this, there is minimal research into the validity of automated tackle detection algorithms and the utility of such devices in quantifying the frequency of collisions in rugby league $[35,37]$.

To our knowledge, only two microtechnology devices from one manufacturer (i.e. Catapult minimaxX and
Optimeye devices) have been validated for automated collision detection in rugby league [35, 37]. The respective devices utilise an algorithmic approach to detect collisions via spikes in instantaneous PlayerLoad (arbitrary units $[\mathrm{AU}])$ and changes in unit orientation that are detected via the gyroscope and magnetometer [31, 35, 37]. The Catapult minimaxX device has been utilised extensively to quantify collisions, with the 2010 validation study in rugby league training receiving over 100 citations to date [31]. Indeed, the authors of this study cite a near perfect correlation between microtechnology detected and video coded collisions $(r=0.96)$ as evidence for the device's automatic collision detection validity [31, 37]. Unfortunately, this approach to validation is potentially problematic as it fails to report a number of factors regarding the precision of this microtechnology for the detection of collision events. More specifically, a) the sensitivity of the device (i.e. truepositive and false-negative ratio); b) the ability of the device to not report collision events, when they do not occur (i.e. specificity; true-negative and false-positive ratio); and c) the accuracy of the device to identify collisions within rugby league match-play, have not been elucidated [35]. Indeed, whilst there is a strong overall relationship between video coded and microtechnology derived collisions, discrepancies in the relationship are observed, particularly with players who undertake fewer collisions (see Fig. 1 in [31]). Additionally, this research did not report the post-collection data processing that was undertaken, which is important for its reproducibility and usability. Collectively, this suggests that this device has yet to be appropriately validated for automated collision detection. As such, until the minimaxX device has been appropriately validated, practitioners should exercise caution when utilising this device in isolation (i.e. without video analysis of collisions) to quantify the frequency of collisions in field-based team sports.

The Catapult Optimeye S5 device has recently undergone validation for the collision detection algorithm utilising a criterion validity framework. Hulin et al. [35] compared microtechnology detected collisions to video coded collisions as a criterion measure during rugby league match-play. In this context, the true-positive was reported when a player was involved in a collision and the device recorded that collision, whilst a false-positive was reported when the player was not involved in a collision and the microtechnology device recorded a collision. Conversely, a false-negative was reported when the player was involved in a collision and the device did not record the collision, and a true-negative was reported when 1) the microtechnology device recorded a $>2 \mathrm{AU}$ PlayerLoad spike; 2) the player was not involved in a collision; and 3) the microtechnology device did not report a collision [35]. Following removal of short duration (< $2 \mathrm{~s}$ ) and low intensity ( $<1$ PlayerLoad AU) events, it was 
reported that the ability of the device to not report collision events when they do not occur (i.e. specificity) was $91.7 \%$, and the ability to detect a collision when it did occur (i.e. sensitivity) was $97.6 \%$ [35]. The accuracy improved to $92.7 \%$ following the removal of short duration and low intensity events during data processing, with the majority of false positives identified as being related to rapid change of directions. Whilst post-collection processing of the data to remove errors may be considered a limitation, this information enables applied practitioners to attain a similar level of accuracy when monitoring collisions via microtechnology. Indeed collisions of low duration and intensity may not be as physically or perceptually fatiguing as those of higher durations and intensities [27]. As such, it may be less pertinent to consider these collisions in the context of contact load monitoring. Given the limited validation research of collision frequency detection, further research investigating the validity of commercially available devices to quantify collision events is warranted [4].

Research that has attempted to explore collision intensity via microtechnology exhibits similar limitations. Specifically, in the original work describing subjective intensity classification [31], it is unclear how the device is able to automate the categorisation of collisions based on subjective intensity (i.e. mild, moderate, heavy). From this study [31] it appears that the respective microtechnology device may not have the capacity to automate collision load monitoring, as the resulting analysis only identifies that the frequency of collisions is highly correlated to the number of collisions in each qualitative intensity descriptor $(r=0.89$, 0.97 and 0.99 for mild, moderate and heavy collisions, respectively). As outlined previously this approach does not identify the number of collisions that were or were not classified correctly using appropriate validity statistics (i.e. specificity, sensitivity, accuracy). A later study included microtechnology-based $g$ forces alongside the qualitative descriptors of mild (1-2g), moderate (2.1-4 $g$ ), and heavy $(>4 g)$ [31, 73]. Whether these $g$ forces and associated zones represent the same zones as the original research is however, unclear. Furthermore, the $g$ forces associated with these zones are notably lower than the highest $g$ force zones reported by other microtechnology devices and studies (see Table 5). This discrepancy highlights differences in microtechnology devices both within and between manufacturers with respect to hardware differences (i.e. inertial sensors, sampling frequency of both GPS and inertial sensors, and dissimilar chipset configurations) [10]. Given these disparities, it is not possible to generalise the validity or reliability-based findings of one device to that of another. With this in mind, any device or algorithm that is launched commercially needs to be appropriately validated against criterion measures, even if they are considered to be iterations of currently available and validated hardware, software or algorithms (i.e. Catapult minimaxX and Optimeye devices). Until devices and algorithms have undergone appropriate external validation practitioners and researchers alike should be cautious in interpreting the reported collision frequency or intensity information. This knowledge is important for practitioners to be cognisant of when selecting and using microtechnology.

Despite representing a relatively small portion of overall match-play time, collisions are one of the most physically demanding aspects of rugby league [26]. With the increasing availability of microtechnology devices and their proliferation in rugby league, both research and practice has shifted to an increasing reliance upon these devices to quantify every aspect of training and competition $[4,9,74]$. This shift has led to further innovation and automation in the approaches utilised to quantify collisions. Recently, a novel metric that purports to combine accelerometer impact forces, velocity into contact, and the collision duration has been developed by STATSports (STATSports, Northern Ireland) [70]. Developed utilising data from rugby union, this collision load metric is described as a "... weighted score providing an intensity of each collision..." [70]. Beyond this, specific information in regards to the algorithm and validation of the metric is currently lacking outside of rugby union [70]. One challenge that remains in the validation of microtechnology to appropriately quantify collision intensity is the lack of an appropriate criterion to validate device metrics against. Whilst microtechnology collision frequency can be compared to video-based methods to establish criterion validity, currently no criterion measure exists in order to validate collision intensity. Indeed, various methods to quantify physical or collision workload intensity have been suggested in the research including subjective measures such as rating of perceived challenge [75], rating of perceived exertion (RPE) [76], and rating of mental effort [76]. Others have investigated intensity based on objective markers including muscle damage biomarkers (such as creatine kinase) [27], and shoulder impact forces [77]. As there is currently no gold standard and a lack of consensus in regard to the measure(s) that appropriately capture collision intensity, future research into the validation of collision intensity and load metrics as reported via microtechnology is warranted. Such metrics may provide further insight into the monitoring of training and game loads, injury prevention, and physical conditioning [4]. Despite this, no microtechnology device to date has the ability to differentiate between collisions, tackles and ball carries, or other sports specific actions (missed tackles, scoring a try, offloads etc.) which involve collisions in rugby league [4]. In other sports, recent research has shown the promise of machine learning methods to automate collision event detection $[34,78,79]$. This research indicated 
that utilising microtechnology data in rugby union, machine learning approaches such as random forests and decision trees can accurately detect and quantify sportsspecific actions, such as scrummaging [78], one-on-one tackling and rucks [34]. It's clear that these approaches can distinguish between different features in large and complex, noisy datasets such as those regularly recorded from microtechnology in sport. Given the potential of such data processing methods, the application of machine learning methods to microtechnology data from rugby league may enable the ability to differentiate collision events (e.g. tackles and ball carries).

\section{Limitations}

A limitation of existing literature is that studies have utilised dissimilar approaches to grouping individual positions to various groups and subgroups. This has meant that there is a general lack of consistency across studies, with a number of studies utilising positional groupings that haven't been replicated by later research. This lack of consistency may have contributed to the high heterogeneity that was present in the collision demands derived from microtechnology in the various groups.

Similarly, there is a lack of consistency regarding approaches to quantifying collision intensity from microtechnology. Studies have utilised a variety of different $g$ force zones or subjective intensity descriptors that differ both within and between devices and manufacturers (see Tables 5 and 6). Furthermore, a number of studies in the review did not report the number of participants in each cohort, or the number of microtechnology files that precluded their inclusion in the current meta-analysis. As such, as has previously been suggested [15], a consensus on the definition of positional groups, zone intensities and descriptors for both impact metrics and accelerometer loads are required to facilitate comparisons within individual sporting codes and levels of participation.

\section{Future directions}

Although microtechnology has been comprehensively adopted in male senior rugby league, independent validation of microtechnology in the detection of collisions is needed, as a number of current devices and algorithms have not been sufficiently externally validated. This is potentially problematic as practitioners may be using these devices with misplaced confidence in their ability to automate collision detection. Similarly, validation of microtechnology collision load metrics and their constituents against the physical force of collisions is warranted, as such information may be of interest to practitioners. Indeed, a study by Usman et al. [77] has investigated the forces of tackles in rugby union athletes using a static instrumented tackle bag. Peak impact force progressively declined with increasing levels of fatigue, and lower forces were observed in the non-dominant shoulder when compared to the dominant shoulder [77]. Further research that investigates aspects of the collision in dynamic situations has just begun to emerge in the wider research [80], and these models hold promise for the investigation of microtechnology collision intensity validation.

If researchers and practitioners are to continue to glean information from microtechnology devices using $g$ force intensity zones, or qualitative descriptors, then they must be aware of the limitations of these approaches as highlighted within this review. Similarly, they should understand that due to inherent differences in the devices and associated algorithms, they are unable to generalise their data across different microtechnology devices and manufacturers. Each new device and detection algorithm that enters the market should be validated against criterion measures. This applies to iterations of previously validated devices.

Currently microtechnology cannot appropriately quantify the post-contact wrestle phase of the collision, which is considered a highly fatiguing aspect of match-play [26]. To address this limitation, a move to utilising sophisticated analytic methods (such as machine learning), and a mechanical model to quantify these actions through the work-energy theorem has recently been suggested [71]. By applying these methods to microtechnology data, the relative contribution of locomotor and collision loads may be able to be partitioned and approximated appropriately. Whilst such approaches hold promise to collision modelling, they have yet to be fully elucidated.

Machine learning approaches have recently been utilised in a range of other sports for their ability to differentiate sports-specific actions in complex and noisy microtechnology datasets [34, 78, 81]. Investigating whether machine learning methods can differentiate between collision-based events such as tackles and ball carries from the overall collision pool is warranted. Automating this process would streamline analysis and provide practitioners with further detailed information on contact loads. This would inform short-, and long-term collision load monitoring, and allow for the exploration of interactions with contact-related injuries [31], contact adaptation [27], and the effects of contact skill and conditioning programs $[4,82]$.

\section{Conclusions}

The quantification of collisions has transitioned from video notational analysis methods to the use of microtechnology devices and associated algorithms to quantify both collision frequency and intensity. Differential collision profiles have been observed in the literature between forward and back positional groups, and their distinct subgroups. The hit-up and tackle demands of forwards and backs differ with forwards experiencing an increased absolute and relative frequency of tackles and collisions. 
Microtechnology has been utilised to quantify collision frequency and intensity but a number of disparate approaches have been undertaken with little consensus to an ideal approach having been established. Furthermore, despite widespread popularity, a number of the microtechnology devices have not been appropriately validated for use in rugby league. Future research using microtechnology should establish the criterion validity of current and novel devices with collision detection algorithms in measuring collision frequency. Similarly, future research should look to establish the measures that capture the intensity of collisions and examine the relationship between collision intensity metrics and directly assessed impact forces. Examining whether machine learning approaches can differentiate between collision-based events such as tackle and ball carry actions are warranted.

\section{Abbreviations}

Cl: Confidence interval; GPS: Global positioning systems; Hz: Samples per second; NR: Not reported; NRL: National Rugby League; NSWCup: New South Wales Cup Competition; NYC: Australian National Under 20's Youth Competition; QCup: Queensland Cup Competition; SL: Super League

\section{Acknowledgements}

Not applicable.

\section{Authors' contributions}

All authors contributed to the conception and design of the review. MN, and CC contributed to the completion of the search strategy and extraction of the relevant data. MN was responsible for the meta-analysis. All authors were involved in the development of the manuscript drafted by MN. The co-authors read and approved the final corrected manuscript as opposed to the original publication.

\section{Funding}

This research received no specific grant from any funding agency in the public, commercial or not-for-profit sectors. The National Rugby League (NRL) provided support in the form of research funding for Cloe Cummins but played no role in the study design, data collection and analysis, decision to publish or preparation of the manuscript.

\section{Availability of data and materials}

Not applicable.

\section{Ethics approval and consent to participate}

Not applicable.

\section{Consent for publication}

Not applicable.

\section{Competing interests}

Mitchell Naughton, Ben Jones, Sharief Hendricks, Doug King, Aron Murphy and Cloe Cummins have no conflicts of interest that are directly relevant to the content of this article. Cloe Cummins has previously held employment with a microtechnology manufacturer. Mitch Naughton's PhD is being undertaken in collaboration with a microtechnology company (STATSports Technologies Ltd.). This company played no role in the study design, data collection and analysis, decision to publish or preparation of the manuscript.

\section{Author details}

'School of Science and Technology, University of New England, Armidale, NSW, Australia. ${ }^{2}$ Carnegie Applied Rugby Research (CARR) Centre, Institute for Sport Physical Activity and Leisure, Leeds Beckett University, Leeds, UK. ${ }^{3}$ Leeds Rhinos Rugby League Club, Leeds, UK. ${ }^{4}$ England Performance Unit, The Rugby Football League, Leeds, UK. ${ }^{5}$ Division of Exercise Science and Sports Medicine, Department of Human Biology, Faculty of Health Sciences, University of Cape Town, Cape Town, South Africa. ${ }^{6}$ Health through Physical
Activity, Lifestyle and Sport Research Centre (HPALS), Faculty of Health Sciences, the University of Cape Town, Cape Town, South Africa. 'Sports Performance Institute New Zealand (SPRINZ), Faculty of Health and Environmental Science, Auckland University of Technology, Auckland, New Zealand. ${ }^{8}$ Traumatic Brain Injury Network (TBIN), Auckland University of Technology, Auckland, New Zealand. ${ }^{9}$ National Rugby League, Sydney, Australia.

\section{1-2.}

\section{References}

1. Woods C, Leicht A, Jones B, Till K. Game-play characteristics differ between the European super league and the National Rugby League: implications for coaching and talent recruitment. Int J Sports Sci Coach. 2018;13(6):1171-6.

2. Gerrard B. Is the Moneyball approach transferable to complex invasion team sports? Int J Sport Financ. 2007;2(4):214

3. Twist C, Highton J, Waldron M, Edwards E, Austin D, Gabbett T. Movement demands of elite rugby league players during Australian National Rugby League and European super league matches. Int J Sports Physiol Perform. 2014:9(6):925-30

4. Hausler J, Halaki M, Orr R. Application of global positioning system and microsensor Technology in Competitive Rugby League Match-Play: a systematic review and meta-analysis. Sports Med. 2016;46(4):559-88.

5. Waldron M, Twist C, Highton J, Worsfold P, Daniels M. Movement and physiological match demands of elite rugby league using portable global positioning systems. J Sports Sci. 2011;29(11):1223-30.

6. Delaney J, Thornton H, Duthie G, Dascombe B. Factors that influence running intensity in interchange players in professional Rugby league. Int J Sports Physiol Perform. 2016;11(8):1047-52.

7. Weaving D, Sawczuk T, Williams S, Scott T, Till K, Beggs C, et al. The peak duration-specific locomotor demands and concurrent collision frequencies of European super league rugby. J Sports Sci. 2019;37(3):322-30.

8. Dempsey G, Gibson N, Sykes D, Pryjmachuk B, Turner A. Match demands of senior and junior players during international Rugby league. J Strength Cond Res. 2018:32(6):1678-84.

9. Johnston RD, Gabbett TJ, Jenkins DG. Applied sport science of rugby league. Sports Med. 2014:44(8):1087-100.

10. Malone J, Lovell R, Varley M, Coutts A. Unpacking the Black box: applications and considerations for using GPS devices in sport. Int I Sports Physiol Perform. 2017;12:S218-s26.

11. Kaplan E, Hegarty C. Understanding GPS: principles and applications: Artech house; 2005.

12. Steede-Terry K, Dangermond J. Integrating GIS and the global positioning system: ESRI, Inc.; 2000.

13. Schutz Y, Chambaz A. Could a satellite-based navigation system (GPS) be used to assess the physical activity of individuals on earth? Eur J Clin Nutr. 1997;51(5):338.

14. Scott M, Scott T, Kelly V. The validity and reliability of global positioning systems in team sport: a brief review. J Strength Cond Res. 2016:30(5):1470-90.

15. Cummins C, Orr R, O'Connor H, West C. Global positioning systems (GPS) and microtechnology sensors in team sports: a systematic review. Sports Med. 2013;43(10):1025-42.

16. Varley M, Gabbett T, Aughey R. Activity profiles of professional soccer, rugby league and Australian football match play. I Sports Sci. 2014; 32(20):1858-66.

17. Scott B, Lockie R, Knight T, Clark A. Janse de Jonge X. a comparison of methods to quantify the in-season training load of professional soccer players. Int J Sports Physiol Perform. 2013;8(2):195-202.

18. Buchheit M, Manouvrier C, Cassirame J, Morin J. Monitoring locomotor load in soccer: is metabolic power, powerful? Int J Sports Med. 2015;36(14):1149-55.

19. Abbott W, Brickley G, Smeeton NJ. Positional differences in GPS outputs and perceived exertion during soccer training games and competition. J Strength Cond Res. 2018;32(11):3222-31.

20. Wisbey B, Montgomery P, Pyne D, Rattray B. Quantifying movement demands of AFL football using GPS tracking. J Sci Med Sport. 2010;13(5):531-6.

21. Brewer C, Dawson B, Heasman J, Stewart G, Cormack S. Movement pattern comparisons in elite (AFL) and sub-elite (WAFL) Australian football games using GPS. J Sci Med Sport. 2010;13(6):618-23.

22. Cunniffe B, Proctor W, Baker J, Davies B. An evaluation of the physiological demands of elite rugby union using global positioning system tracking software. J Strength Cond Res. 2009;23(4):1 195-203. 
23. Coughlan G, Green B, Pook P, Toolan E, O'connor S. Physical game demands in elite rugby union: a global positioning system analysis and possible implications for rehabilitation. J Orth Sports Phys Ther. 2011;41(8):600-5.

24. Gabbett T. Influence of playing standard on the physical demands of professional rugby league. J Sports Sci. 2013;31(10):1125-38.

25. Gabbett $T$, Jenkins D, Abernethy B. Physical demands of professional rugby league training and competition using microtechnology. J Sci Med Sport. 2012;15(1):80-6.

26. Cummins C, Orr R. Analysis of physical collisions in elite National Rugby League Match Play. Int J Sports Physiol Perform. 2015;10(6):732-9.

27. Naughton M, Miller J, Slater G. Impact-induced muscle damage and contact sports: etiology, effects on neuromuscular function and recovery, and the modulating effects of adaptation and recovery strategies. Int J Sports Physiol Perform. 2018;13(8):962-9.

28. King $D$, Hume $P$, Clark T. Nature of tackles that result in injury in professional Rugby league. Res Sports Med. 2012;20(2):86-104.

29. Parmar N, James N, Hearne G, Jones B. Using principal component analysis to develop performance indicators in professional rugby league. Int J Perform Anal Sport. 2018;18(6):938-49.

30. Woods C, Sinclair W, Robertson S. Explaining match outcome and ladder position in the National Rugby League using team performance indicators. J Sci Med Sport. 2017;20(12):1107-11.

31. Gabbett $T$, Jenkins D, Abernethy B. Physical collisions and injury during professional rugby league skills training. J Sci Med Sport. 2010;13(6):578-83.

32. Sirotic A, Coutts A, Knowles H, Catterick C. A comparison of match demands between elite and semi-elite rugby league competition. J Sports Sci. 2009;27(3):203-11.

33. Gabbett T. Influence of fatigue on tackling technique in rugby league players. J Strength Cond Res. 2008;22(2):625-32.

34. Chambers R, Gabbett T, Gupta R, Josman C, Bown R, Stridgeon P, et al. Automatic detection of one-on-one tackles and ruck events using microtechnology in rugby union. J Sci Med Sport. 2019;22:827-32.

35. Hulin B, Gabbett T, Johnston R, Jenkins D. Wearable microtechnology can accurately identify collision events during professional rugby league matchplay. J Sci Med Sport. 2017;20(7):638-42

36. Gabbett T, Hulin B. Activity and recovery cycles and skill involvements of successful and unsuccessful elite rugby league teams: a longitudinal analysis of evolutionary changes in National Rugby League match-play. J Sports Sci. 2018;36(2):180-90

37. Gabbett T. Quantifying the physical demands of collision sports: does microsensor technology measure what it claims to measure? J Strength Cond Res. 2013;27(8):2319-22.

38. McLellan C, Lovell D, Gass G. Biochemical and endocrine responses to impact and collision during elite Rugby league match play. J Strength Cond Res. 2011;25(6):1553-62.

39. Rohatgi A. WebPlotDigitizer; 2011.

40. Downs S, Black N. The feasibility of creating a checklist for the assessment of the methodological quality both of randomised and non-randomised studies of health care interventions. J Epidemiol Community Health. 1998;52(6):377-84.

41. King D, Hume P, Clark T. Video analysis of tackles in professional rugby league matches by player position, tackle height and tackle location. Int J Perform Anal Sport. 2010;10(3):241-54.

42. Kempton T, Sirotic A, Coutts A. A comparison of physical and technical performance profiles between successful and less-successful professional Rugby league teams. Int J Sports Physiol Perform. 2017;12(4):520-6.

43. Higgins JP, Green S. Cochrane handbook for systematic reviews of interventions: Wiley; 2011.

44. Gabbett T, Jenkins D, Abernethy B. Physical collisions and injury in professional rugby league match-play. J Sci Med Sport. 2011;14(3):210-5.

45. Borenstein M, Hedges LV, Higgins JP, Rothstein HR. Introduction to metaanalysis: Wiley; 2011.

46. Austin D, Gabbett T, Jenkins D. Tackling in professional rugby league. J Strength Cond Res. 2011;25(6):1659-63.

47. Gabbett T. Relationship between accelerometer load, collisions, and repeated high-intensity effort activity in Rugby league players. J Strength Cond Res. 2015:29(12):3424-31.

48. Gabbett T. Influence of ball-in-play time on the activity profiles of rugby league match-play. J Strength Cond Res. 2015;29(3):716-21.

49. Gabbett T, Jenkins D, Abernethy B. Relationships between physiological, anthropometric, and skill qualities and playing performance in professional rugby league players. J Sports Sci. 2011;29(15):1655-64.
50. Gabbett T, Seibold A. Relationship between tests of physical qualities, team selection, and physical match performance in semiprofessional rugby league players. J Strength Cond Res. 2013;27(12):3259-65.

51. Gabbett T, Stein J, Kemp J, Lorenzen C. Relationship between tests of physical qualities and physical match performance in elite rugby league players. J Strength Cond Res. 2013;27(6):1539-45.

52. Hulin B, Gabbett T, Kearney S, Corvo A. Physical demands of match play in successful and less-successful elite Rugby league teams. Int J Sports Physiol Perform. 2015;10(6):703-10.

53. Johnston R, Gibson N, Twist C, Gabbett T, MacNay S, MacFarlane N. Physiological responses to an intensified period of rugby league competition. J Strength Cond Res. 2013;27(3):643-54.

54. Kempton T, Sirotic A, Coutts A. An integrated analysis of match-related fatigue in professional rugby league. J Sports Sci. 2015;33(1):39-47.

55. Lovell T, Sirotic A, Impellizzeri F, Coutts A. Factors affecting perception of effort (session rating of perceived exertion) during Rugby league training. Int J Sports Physiol Perform. 2013;8(1):62-9.

56. McLellan C, Lovell D. Neuromuscular responses to impact and collision during elite rugby league match play. J Strength Cond Res. 2012;26(5):1431-40.

57. Murray N, Gabbett T, Chamari K. Effect of different between-match recovery times on the activity profiles and injury rates of national rugby league players. J Strength Cond Res. 2014;28(12):3476-83.

58. Oxendale C, Twist C, Daniels M, Highton J. The relationship between matchplay characteristics of elite Rugby league and indirect markers of muscle damage. Int J Sports Physiol Perform. 2016;11(4):515-21.

59. Sirotic A, Knowles H, Catterick C, Coutts A. Positional match demands of professional rugby league competition. J Strength Cond Res. 2011;25(11): 3076-87.

60. Cummins C, Orr R. Collision characteristics of shoulder charge tackles in elite rugby league. Int J Perform Anal Sport. 2015;15(3):1090-101.

61. Speranza M, Gabbett T, Greene D, Johnston R, Townshend A. Relationship between 2 standardized tackling proficiency tests and Rugby league match-play tackle performance. Int J Sports Physiol Perform. 2018;13(6):770-6.

62. Twist C, Waldron M, Highton J, Burt D, Daniels M. Neuromuscular, biochemical and perceptual post-match fatigue in professional rugby league forwards and backs. J Sports Sci. 2012;30(4):359-67.

63. Weaving D, Marshall P, Earle K, Nevill A, Abt G. Combining internal-and external-training-load measures in professional rugby league. Int J Sports Physiol Perform. 2014:9(6):905-12.

64. Woods C, Robertson S, Sinclair W, Till K, Pearce L, Leicht A. A comparison of game-play characteristics between elite youth and senior Australian National Rugby League competitions. J Sci Med Sport. 2018;21(6):626-30.

65. Evans S, Brewer C, Haigh J, Lake M, Morton J, Close G. The physical demands of super league rugby: experiences of a newly promoted franchise. Eur J Sport Sci. 2015;15(6):505-13.

66. Fletcher B, Twist C, Haigh J, Brewer C, Morton J, Close G. Season-long increases in perceived muscle soreness in professional rugby league players: role of player position, match characteristics and playing surface. J Sports Sci. 2016:34(11):1067-72

67. Gabbett T, Ryan P. Tackling technique, injury risk, and playing performance in high-performance collision sport athletes. Int J Sports Sci Coach. 2009;4(4):521-33.

68. Gissane C, Jennings D, Jennings S, White J, Kerr K. Physical collisions and injury rates in professional super league rugby. Cleve Med J. 2001;4:147-55.

69. Hendricks S, Karpul D, Lambert M. Momentum and kinetic energy before the tackle in rugby union. J Sports Sci Med. 2014;13(3):557-63.

70. MacLeod S, Hagan C, Egana M, Davis J, Drake D. The use of microtechnology to monitor collision performance in professional Rugby union. Int J Sports Physiol Perform. 2018;13(8):1075-82.

71. Gray A, Shorter K, Cummins C, Murphy A, Waldron M. Modelling movement energetics using global positioning system devices in contact team sports: limitations and solutions. Sports Med. 2018;48(6):1357-68.

72. McLean B, Cummins C, Conlan G, Duthie G, Coutts A. The fit matters: influence of accelerometer fitting and training drill demands on load measures in rugby league players. Int J Sports Physiol Perform. 2018;13(8):1083-9.

73. Gabbett T, Polley C, Dwyer D, Kearney S, Corvo A. Influence of field position and phase of play on the physical demands of match-play in professional rugby league forwards. J Sci Med Sport. 2014;17(5):556-61.

74. Hulin B, Gabbett T, Johnston R, Jenkins D. PlayerLoad variables: sensitive to changes in direction and not related to collision workloads in Rugby leaque match play. Int J Sports Physiol Perform. 2018;13(9):1136-42. 
75. Hendricks S, Till K, Oliver J, Johnston R, Attwood M, Brown J, et al. Rating of perceived challenge as a measure of internal load for technical skill performance. Br J Sports Med. 2019;53(10):611-3.

76. Hendricks S, Till K, Oliver J, Johnston R, Attwood M, Brown J, et al. Technical skill training framework and skill load measurements for the rugby union tackle. J Strength Cond Res. 2018;40(5):44-59.

77. Usman J, Mclntosh A, Frechede B. An investigation of shoulder forces in active shoulder tackles in rugby union football. J Sci Med Sport. 2011;14(6):547-52.

78. Chambers R, Gabbett T, Cole M. Validity of a microsensor-based algorithm for detecting scrum events in Rugby union. Int I Sports Physiol Perform. 2019:14(2):176-82.

79. Kelly D, Coughlan G, Green B, Caulfield B. Automatic detection of collisions in elite level rugby union using a wearable sensing device. Sports Eng. 2012;15(2):81-92.

80. Burger $\mathrm{N}$, Lambert M, Hall H, Hendricks S. Assessing tackle performance using a novel collision sport simulator in comparison to a "live" one-on-one tackling drill. J Sports Sci. 2018:1-8.

81. Wundersitz D, Josman C, Gupta R, Netto K, Gastin P, Robertson S. Classification of team sport activities using a single wearable tracking device. J Biomech. 2015;48(15):3975-81.

82. Hendricks S, Till K, Brown J, Jones B. Rugby union needs a contact skilltraining programme. Br J Sports Med. 2017:829-30.

\section{Publisher's Note}

Springer Nature remains neutral with regard to jurisdictional claims in published maps and institutional affiliations.

\section{Submit your manuscript to a SpringerOpen ${ }^{\circ}$ journal and benefit from:}

- Convenient online submission

- Rigorous peer review

- Open access: articles freely available online

- High visibility within the field

- Retaining the copyright to your article

Submit your next manuscript at $\boldsymbol{\nabla}$ springeropen.com 